\title{
Modelling, control and Pontryagin Maximum Principle for a two-body wave energy device
}

\author{
José J. Cândido ${ }^{1}$, Paulo A.P.S. Justino ${ }^{2}$ \\ ${ }^{1}$ IDMEC, Instituto Superior Técnico, Technical University of Lisbon \\ Av. Rovisco Pais, 1049-001 Lisboa, Portugal \\ E-mail address: jose.candido@ist.utl.pt \\ ${ }^{2}$ Renewable Energies Department, INETI/LNEG \\ Estrada do Paço do Lumiar, 1649-038 Lisbon, Portugal
}

\begin{abstract}
Frequency domain analysis is applied to a wave energy device composed by two coaxial axisymmetric bodies. For each frequency optimal damping coefficient values which maximize absorbed power are obtained. Several displacement amplitude restriction scenarios are considered. A stochastic model to describe the device's behaviour in irregular waves is developed. Optimal mechanical damping and spring coefficients are computed. Considering different sea state conditions, probability density functions are defined for relevant parameters and time averaged absorbed power values are obtained.

A time domain model is also developed for the device. A non-linear power take-off mechanism configuration, consisting in a hydraulic circuit with low-pressure and high-pressure gas accumulators, is devised. Time averaged absorbed power is maximized in terms of characteristic mechanism parameter. A sub-optimal method of phase control by latching is applied to the device in order to improve its performance. Analytical development of Pontryagin Maximum Principle is used to establish an algorithm for device's control.
\end{abstract}

Keywords: stochastic modelling; time domain modelling; Pontryagin Maximum Principle; twobody device.

\section{Introduction}

Wave energy devices design and development must necessarily follow several stages prior to their full scale construction and real sea deployment. Standard procedure should account for an initial step including the theoretical study, mathematical modelling as well as numerical simulation of the device behaviour. This should be followed by physical testing at reduced scale 
in a wave basin or flume, and a final stage, previous to the definitive installation, comprising prototype sea tests at full, or smaller than full, scale.

Mathematical modelling and numerical simulation are essential not only for the preliminary analysis of the device behaviour but also for the optimization of the geometry. Furthermore, the complexity of wave energy utilization, which involves a chain of energy conversion processes, characterized by their own efficiency and by the constrains they introduce, implies the necessity of establishing control strategies to optimize the global performance of the device. Mathematical modelling and numerical simulation constitute the most adequate means for the development and test of these strategies, which should be adapted to the device characteristics. Taking into consideration that linear water wave theory is adopted in this type of modelling, its main limitations reside in the impossibility to account for losses due to viscous effects and turbulence associated to real fluids and to accurately reproduce the device behaviour for large amplitude incident waves.

Offshore devices exploit the higher wave energy resources of deep water sites, allowing array dispositions of several units which considerably attenuate the characteristic high transferred power fluctuations. Furthermore, this type of devices reduces substantially the visual impact verified in the case of shoreline and near-shore devices. In an important class of the currently existing (or planned) offshore wave devices, the energy extraction results from the oscillating movement of a single body reacting against a fixed frame of reference (the sea bottom or a bottom-fixed structure). Since the distance between the floating body and the sea bottom may be considerably large in the intended offshore sites, this configuration may lead to practical difficulties. In these circumstances, alternative configurations should be considered, in which the wave energy extraction occurs from the relative oscillating movement between two bodies.

The present discussion refers to a two-body wave energy device. In [1] the theoretical basis for the analysis of the hydrodynamics of such devices is established. The theory for power absorption by two independently one-mode oscillating cylinders was treated in [2]. A comparison between systems of coupled oscillating bodies is made in [3], considering the reaction mass both submerged and on board the extracting body. [4] deals with the linearized hydrodynamic radiation problem for two vertical coaxial cylindrical floaters, independently oscillating in heave in finite depth. In [5] an approach to time domain modelling of multi-body systems is proposed and validated for a two-body system.

In the initial steps of the numerical modelling of a device a frequency domain analysis is frequently used, as in the case of Searev [6]. This applies also to arrays of devices [7]. In the present paper, frequency-domain analysis is initially applied to the two-body wave energy device, consisting of a toroidal buoy and a set of two cylinders, one floating and another completely submerged, rigidly connected to each other. This approach proved to be particularly useful in the tuning of the device for specific frequencies of the spectrum, namely as far as characteristic power take-off coefficients are concerned. 
Subsequently, in order to evaluate the device behaviour in the frequency-domain for irregular waves a stochastic model was developed. Such models were previously developed for OWC power plants [8]. Probability density functions are defined for the relevant parameters that characterize the device behaviour. Assuming that the overall system behaviour is linear and that the wave elevation for irregular waves may be regarded as a stochastic process with a Gaussian probability density function, the variables that define the system behaviour, such as the displacements of the bodies, will also hold a Gaussian probability density function. For these parameters and different sea state conditions the probability density functions are obtained in order to characterize the system. The average power extraction is also computed for these sea state conditions.

Frequency domain analysis does not allow considering non-linear power take-off system configurations, which would be the most realistic scenario for the majority of wave power devices. This difficulty may be overcome by means of time domain analysis. Time domain models allow the computation of time series for the variables that characterize the wave power system behaviour, either assuming a linear or non-linear power take-off system. A time domain model is also developed here for the two-body wave power device. A non-linear power take-off configuration, consisting of a hydraulic circuit with a high-pressure gas accumulator, a lowpressure gas accumulator and a hydraulic motor, as in [9], is considered. The parameter relating the flow rate through the hydraulic motor to the pressure difference between the two accumulators is assumed to be a control parameter. Additionally, some device control strategies are established and tested. The sub-optimal phase control by lacthing strategy proposed by [10], which proved to be efficient in the situation of single-body wave energy converters, is applied to the two-body device.

A control strategy is also established assuming a linear power take-off mechanism. In particular, a control algorithm is developed for the device based on Pontryaguin Maximum Principle [11]. It is concluded that damping control should be of the on-off kind except for singular arcs.

\section{Mathematical models}

Consider the wave energy device represented in Fig. 1, made of two coaxial axisymmetric oscillating bodies: body 1 is the body with a ring like shape (outside body, 10m inside diameter, $13 \mathrm{~m}$ outside diameter, $5.75 \mathrm{~m}$ draught) and body 2 is the set of two cylinders, one surfacepiercing (8m diameter, $8.4 \mathrm{~m}$ draught) and the other completely submerged (10m diameter, $26.9 \mathrm{~m}$ length), $6.2 \mathrm{~m}$ apart and rigidly connected to each other. The extraction of power from the sea waves results from the relative heave motion between bodies. For the purpose of this study it is assumed that the two bodies have linear hydrodynamic behaviour. The mass and volume of the structure connecting the two inside cylinders, as well as the hydrodynamic forces on it, 
are neglected (an alternative approach would be to assume that this mass is included in the mass of the completely submerged cylinder).

Fig. 1. Two perspectives of the panel grid describing the wet surface of the coaxial axisymmetric oscillating bodies in numerical evaluation.

\subsection{Frequency domain model}

Following Newton's second law, assuming single oscillating modes for both bodies, namely heave modes $\xi_{1}$ and $\xi_{2}$, and that the power take-off can be modelled by spring and damping terms proportional to the relative displacement and to the relative velocity between bodies, respectively, the governing equations for the wave energy device may be expressed in the frequency domain by

$$
\begin{aligned}
& -M_{1} \omega^{2} \hat{\xi}_{1}=\left(\omega^{2} A_{11}-i \omega B_{11}\right) \hat{\xi}_{1}+\left(\omega^{2} A_{12}-i \omega B_{12}\right) \hat{\xi}_{2}+F_{\mathrm{D}_{1}}-\rho g S_{1} \hat{\xi}_{1}-\left(K_{\mathrm{L}}+i \omega D_{\mathrm{L}}\right)\left(\hat{\xi}_{1}-\hat{\xi}_{2}\right), \\
& -M_{2} \omega^{2} \hat{\xi}_{2}=\left(\omega^{2} A_{22}-i \omega B_{22}\right) \hat{\xi}_{2}+\left(\omega^{2} A_{21}-i \omega B_{21}\right) \hat{\xi}_{1}+F_{\mathrm{D}_{2}}-\rho g S_{2} \hat{\xi}_{2}+\left(K_{\mathrm{L}}+i \omega D_{\mathrm{L}}\right)\left(\hat{\xi}_{1}-\hat{\xi}_{2}\right) .
\end{aligned}
$$

Here $\omega$ is the angular frequency, $\hat{\xi}_{i}$ the complex amplitude displacement for body $i, M_{i}$ the mass of body $i, \rho$ the water density, $g$ the acceleration of gravity, $S_{i}$ the cross sectional area of body $i$ defined by the undisturbed free-surface, $A_{i j}$ and $B_{i j}$ the added mass and damping hydrodynamic coefficients, $F_{\mathrm{D}_{i}}$ the complex diffraction force amplitude for body $i, K_{\mathrm{L}}$ and $D_{\mathrm{L}}$ the spring and damping coefficients of the power take-off equipment.

According to [12], the time averaged power extracted from a wave with angular frequency $\omega$ is given by

$$
\bar{P}=\frac{1}{2} D_{\mathrm{L}} \omega^{2}\left|\hat{\xi}_{1}-\hat{\xi}_{2}\right|^{2}
$$

The capture width, $\lambda_{c}$, may be obtained from

$$
\lambda_{c}=\frac{\bar{P}}{\bar{P}_{i}}=\frac{2 D_{\llcorner} \omega^{3}\left|\hat{\xi}_{1}-\hat{\xi}_{2}\right|}{\rho g^{2}|\hat{A}(\omega)|^{2}\left(1+\frac{2 k h}{\sinh (2 k h)}\right) \tanh (k h)} \text {, }
$$

where $\bar{P}_{i}$ is the power for an incident regular wave with angular frequency $\omega$ and complex elevation amplitude $\hat{A}(\omega)$. Here $h$ is the water depth and $k$ is the wave number given by the positive root of the dispersion relationship $\omega^{2} / g=k \tanh (k h)$. 


\subsection{Stochastic model}

Similarly to what was done in [8], we assume that the sea surface elevation, $\eta(t)$, is a Gaussian random variable in a time interval $T$, given by

$\eta(t)=\sum_{n=-\infty}^{+\infty} \hat{A}_{n} \exp \left(i n \omega_{0} t\right)$

where $\omega_{0}=2 \pi / T$ and $\hat{A}_{n}=\left|\hat{A}_{n}\right| \exp \left(i \varphi_{n}\right)$ is a complex random variable, $\varphi_{n}$ being a random variable uniformly distributed in the interval $[0,2 \pi[$. Denoting the expected value of a variable by $E\{\}$, it is also assumed that $E\left\{\left|\hat{A}_{n}\right|^{2}\right\}=\sigma_{n}^{2}$ and $E\left\{\hat{A}_{n} \hat{A}_{n^{\prime}}^{*}\right\}=0$, for $n \neq n^{\prime}$.

Assuming that the sea state can be represented by a discrete power spectrum, the variance of the sea surface elevation is defined by [8]

$\sigma_{\eta}^{2}=E\left\{\eta \eta^{*}\right\}=\sum_{n=-\infty}^{+\infty} \sum_{n^{\prime}=-\infty}^{+\infty} \exp \left(i\left(n-n^{\prime}\right) \omega_{0} t\right) E\left\{\hat{A}_{n} \hat{A}_{n}^{*}\right\}=\sum_{n=-\infty}^{+\infty} \sigma_{n}^{2}$

If the power spectrum is continuous the variance of the sea surface elevation is given by

$\sigma_{\eta}^{2}=\int_{-\infty}^{+\infty} S_{\eta}(\omega) d \omega$

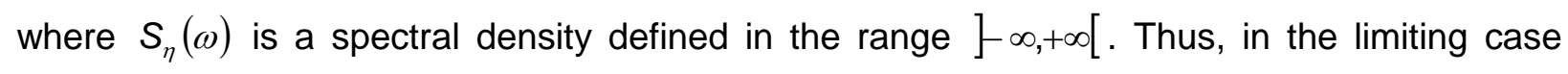
$T \rightarrow \infty$ it will be $\omega_{0} \rightarrow d \omega$ and $E\left\{\left|\hat{A}_{n}\right|^{2}\right\}=\sigma_{n}^{2} \rightarrow S_{\eta}(\omega) d \omega$.

Taking into account Eqs. (1) and (2), which describe the device's behaviour in the frequency domain, and considering that the two oscillating bodies are axisymmetric, it is possible to find transfer functions, $H G_{1}\left(n \omega_{0}\right)$ and $H G_{2}\left(n \omega_{0}\right)$, that relate the amplitude of the incident wave $\hat{A}_{n}$ to the displacement amplitude for body 1 and body 2 , so that

$\hat{\xi}_{1}\left(n \omega_{0}\right)=H G_{1}\left(n \omega_{0}\right) \hat{A}_{n}$

and

$\hat{\xi}_{2}\left(n \omega_{0}\right)=H G_{2}\left(n \omega_{0}\right) \hat{A}_{n}$.

Thus, assuming that (5) holds, the vertical displacements for bodies 1 and 2 are described by

$$
\xi_{1}(t)=\sum_{n=-\infty}^{+\infty} H G_{1}\left(n \omega_{0}\right) \hat{A}_{n} \exp \left(i n \omega_{0} t\right)
$$

and

$\xi_{2}(t)=\sum_{n=-\infty}^{+\infty} H G_{2}\left(n \omega_{0}\right) \hat{A}_{n} \exp \left(i n \omega_{0} t\right)$ 
Note that, in the same manner as $\eta, \xi_{1}$ and $\xi_{2}$ are Gaussian random variables, with variances

$\sigma_{\xi_{1}}^{2}=E\left\{\xi_{1} \xi_{1}^{*}\right\}=\sum_{n=-\infty}^{+\infty}\left|H G_{1}\left(n \omega_{0}\right)\right|^{2} \sigma_{n}^{2}$

and

$\sigma_{\xi_{2}}^{2}=E\left\{\xi_{2} \xi_{2}^{*}\right\}=\sum_{n=-\infty}^{+\infty} \mid H G_{2}\left(n \omega_{0}\right)^{2} \sigma_{n}^{2}$.

In the case of a sea state represented by a continuous power spectrum, the variances of $\xi_{1}$ and $\xi_{2}$ are

$\sigma_{\xi_{1}}^{2}=\int_{-\infty}^{+\infty} S_{\eta}(\omega)\left|H G_{1}(\omega)\right|^{2} d \omega$

and

$\sigma_{\xi_{2}}^{2}=\int_{-\infty}^{+\infty} S_{\eta}(\omega) \mid H G_{2}(\omega)^{2} d \omega$

Assuming that the load force, $F_{\mathrm{L}}$, is given by

$F_{\mathrm{L}}(t)= \pm \sum_{n=-\infty}^{+\infty}\left(K_{\mathrm{L}}+i n \omega_{0} D_{\mathrm{L}}\right)\left(\hat{\xi}_{1}\left(n \omega_{0}\right)-\hat{\xi}_{2}\left(n \omega_{0}\right)\right) \exp \left(i n \omega_{0} t\right)$

using Eqs. (10) and (11) and knowing that $E\left\{\left(\hat{\xi}_{1}-\hat{\xi}_{2}\right)_{n}\left(\hat{\xi}_{1}-\hat{\xi}_{2}\right)_{n^{\prime}}\right\}=0$ for $n \neq n^{\prime}$, the variance of the load force is

$\sigma_{\digamma_{\mathrm{L}}}^{2}=\sum_{n=-\infty}^{+\infty}\left|Z_{\mathrm{L}}\left(n \omega_{0}\right)\right|^{2}\left|H G_{1}\left(n \omega_{0}\right)-H G_{2}\left(n \omega_{0}\right)\right|^{2} \sigma_{n}^{2}$,

where $Z_{\mathrm{L}}\left(n \omega_{0}\right)=K_{\mathrm{L}}+i n \omega_{0} \mathrm{~L}$. For a continuous power spectrum this expression turns into

$\sigma_{F_{\mathrm{L}}}^{2}=\int_{-\infty}^{+\infty} S_{\eta}(\omega)\left|Z_{L}(\omega)^{2}\right| H G_{1}(\omega)-\left.H G_{2}(\omega)\right|^{2} d \omega$

The average absorbed power may be written as

$\bar{P}=D_{\mathrm{L}} E\left\{\left|\dot{\xi}_{1}(t)-\dot{\xi}_{2}(t)\right|^{2}\right\}$

where $\dot{\xi}_{i}$ is the velocity of body $i$ and, in the case of a sea state represented by a continuous power spectrum,

$E\left\{\left|\dot{\xi}_{1}-\dot{\xi}_{2}\right|^{2}\right\}=E\left\{\left(\dot{\xi}_{1}-\dot{\xi}_{2}\right)\left(\dot{\xi}_{1}-\dot{\xi}_{2}\right)^{*}\right\}=\int_{-\infty}^{+\infty} S_{\eta}(\omega) \omega^{2}\left|H G_{1}(\omega)-H G_{2}(\omega)\right|^{2} d \omega$. 


\subsection{Time domain model}

The governing equations (in the time domain) (1) and (2) for the wave energy device take the form

$$
\begin{aligned}
& \left(M_{1}+A_{\infty_{11}}\right) \ddot{\xi}_{1}(t)+A_{\infty_{12}} \ddot{\xi}_{2}(t)+\int_{-\infty}^{t} L_{11}(t-\tau) \ddot{\xi}_{1}(\tau) d \tau+\int_{-\infty}^{t} L_{12}(t-\tau) \ddot{\xi}_{2}(\tau) d \tau+\rho g S_{1} \xi_{1}(t)=f_{\mathrm{D}_{1}}(t)+f_{\mathrm{L}}\left(\xi_{i}, \dot{\xi}_{i}\right), \\
& \left(M_{2}+A_{\infty_{22}}\right) \ddot{\xi}_{2}(t)+A_{\infty_{21}} \ddot{\xi}_{1}(t)+\int_{-\infty}^{t} L_{21}(t-\tau) \ddot{\xi}_{1}(\tau) d \tau+\int_{-\infty}^{t} L_{22}(t-\tau) \ddot{\xi}_{2}(\tau) d \tau+\rho g S_{2} \xi_{2}(t)=f_{\mathrm{D}_{2}}(t)-f_{\mathrm{L}}\left(\xi_{i}, \dot{\xi}_{i}\right) .
\end{aligned}
$$

Here $\ddot{\xi}_{i}$ is the acceleration of body $i, A_{\infty i j}$ is the limiting value, when $\omega \rightarrow \infty$, of the added mass $A_{i j}, f_{\mathrm{D}_{i}}$ the diffraction force on body $i$ and $f_{\mathrm{L}}$ the load force applied on the two bodies by the power take-off equipment, which may be a linear function of the relative velocity and/or relative displacement between bodies or a non-linear function. The convolution integrals introduced in these equations represent the memory effect in the radiation force due to the history of the two bodies' motion [9]. $L_{i j}$ is a memory function, obtained from the hydrodynamic damping coefficient $B_{i j}$ by [12]

$$
L_{i j}(t)=\frac{2}{\pi} \int_{0}^{\infty} \frac{B_{i j}(\omega)}{\omega} \sin \omega t d \omega
$$

A linear configuration for the power take-off mechanism can be devised by representing it as a function of spring and damping terms respectively proportional to the relative displacement between bodies and to the relative velocity. Thus, in this case the load force is given by

$$
f_{\mathrm{L}}\left(\xi_{i}, \dot{\xi}_{i}\right)= \pm K_{\mathrm{L}}\left(\xi_{1}(t)-\xi_{2}(t)\right) \pm D_{\mathrm{L}}\left(\dot{\xi}_{1}(t)-\dot{\xi}_{2}(t)\right) .
$$

The time domain model also allows the representation of a non-linear configuration for the power take-off mechanism, which is the most realistic scenario for the majority of wave power devices. Following [9], we will consider a hydraulic circuit that includes a set of cylinders, highpressure and low-pressure gas accumulators and a hydraulic motor. The relative motion between the two bodies induces the displacement of pistons inside the cylinders. A rectifying valve assures that the liquid always enters the high-pressure accumulator and leaves the lowpressure accumulator, and not otherwise, whether the relative displacement between bodies is made down or upwards. The resulting pressure difference between the accumulators, $\Delta p_{\text {acs }}$, drives the hydraulic motor. It is here adopted the control algorithm proposed by [10] which consists in establishing a proportionality relationship between the instantaneous flow rate through the hydraulic motor, $q_{m}$, and the instantaneous pressure difference between the accumulators, so that

$q_{m}(t)=S_{c}{ }^{2} G_{m} \Delta p_{a c s}(t)$, 
where $G_{m}$ is a constant and $S_{c}$ the total effective cross-sectional area of the pair or pairs of cylinders.

Following [9], the pressure difference between the accumulators is, in turn, obtained from

$$
\Delta p_{a c s}(t)=\Theta_{1} v_{1}(t)^{-\gamma}-\Theta_{2}\left(\frac{v_{0}-m_{1} v_{1}(t)}{m_{2}}\right)^{-\gamma},
$$

where $\Theta_{1}$ and $\Theta_{2}$ are constants for fixed entropy (an isentropic process is assumed) referring to the high-pressure and low-pressure accumulators, respectively, $\gamma=c_{p} / c_{v}$ is the specificheat ratio for the gas inside the accumulators, $m_{1}$ and $m_{2}$ are the masses of gas inside the high-pressure and the low-pressure accumulators, respectively, assumed to be fixed along the process, $v_{1}$ is the specific volume of gas inside the high-pressure accumulator and $V_{0}$ the total volume of gas inside the accumulators, which remains constant along the process, so that $m_{1} v_{1}(t)+m_{2} v_{2}(t)=V_{0}=$ constant $\left(v_{2}\right.$ is the specific volume of gas inside the low-pressure accumulator).

The net flow rate of oil that enters the high pressure accumulator is equal (with the opposite sign) to the rate of change of the gas volume inside the accumulator, that is

$$
q(t)-q_{m}(t)=-m_{1} \frac{d v_{1}(t)}{d t}
$$

where $q$ is the volume flow rate of liquid displaced by the pistons.

In this configuration, the instantaneous power available to the hydraulic motor at a given instant, $P_{m}$, is given by

$P_{m}(t)=q_{m}(t) \cdot \Delta p_{a c s}(t)$

Over a sufficiently long time interval, the time-averaged absorbed power and the time averaged value of $P_{m}$ are identical if energy losses in the hydraulic circuit are ignored.

\section{Frequency domain results}

\subsection{Regular waves}

Frequency-domain analysis in regular waves can be particularly useful in the tuning of the device to specific frequencies, assumed to be representative of the typical wave spectrum for the intended deployment site. If the device performs efficiently in regular waves over a relatively large frequency bandwidth, then it should be expected to operate efficiently in irregular waves with a spectral distribution spanning the same frequency range. Additionally, if linear reactive 
control, simulated by linear mechanical damping and stiffness, is to be considered, it is possible to evaluate the response in frequency of the corresponding mechanical coefficients, hence enabling a more adequate planning of control strategies for irregular waves conditions.

Using WAMITC, hydrodynamic diffraction and radiation coefficients for a set of 441 wave frequencies in the range of $0.15 \mathrm{rad} / \mathrm{s}$ to $2.0977 \mathrm{rad} / \mathrm{s}$ were obtained for the device represented in Fig. 1. An $80 \mathrm{~m}$ water depth was considered.

In the linear configuration initially adopted for the power take-off equipment it is assumed that it can be simulated solely by a damping term $\left(K_{\mathrm{L}}=0\right)$. The values $D_{\mathrm{L}}$ that maximize the absorbed power, hence the capture width, $\lambda_{c}$, were computed.

The results obtained refer to $1 \mathrm{~m}$ amplitude incident waves. In order to avoid unrealistic solutions (body oscillation amplitudes not small compared with body dimensions) that fall out of the scope of linear hydrodynamic theory, several restriction scenarios were considered with respect to the amplitude of the heave motion of body 1 and the amplitude of the relative heave motion between the two bodies, $\left|\hat{\xi}_{1}-\hat{\xi}_{2}\right|$. Fig. 2 presents the dimensionless absorbed power, defined by $\bar{P}^{*}=\bar{P} / \bar{P}_{\max }$, where $\bar{P}$ is given by Eq. (3) and $\bar{P}_{\max }$ is the theoretical maximum limit of the time-averaged power that an axisymmetric heaving wave energy converter can absorb from regular waves with frequency $\omega$ and amplitude $A_{w}, \bar{P}_{\max }=\rho g^{3} A_{w}{ }^{2} /\left(4 \omega^{3}\right)$ [12]. The different curves refer to the different displacement amplitude scenarios, so that the first number in the legend corresponds to the maximum relative vertical displacement ( $5 \mathrm{~m}$ in every case) and the second to the maximum absolute vertical displacement for body 1 ( $4 \mathrm{~m}$ to $8 \mathrm{~m}$ ). Two clear peaks are observed in the figure, the first one for wave periods around $T=5.5 \mathrm{~s}$ and the second for wave periods close to $T=11.2 \mathrm{~s}$. Time-averaged absorbed power clearly depends on the maximum amplitude allowed for body 1 vertical displacement in the second peak, so that higher $\bar{P}^{*}$ values correspond to higher maximum amplitude values. As a consequence of the amplitude being restricted for these wave periods, approximately only $63 \%$ of the theoretical limit $\bar{P}_{\max }(\bar{P} \approx 875 \mathrm{~kW})$ is reached, if a $8 \mathrm{~m}$ maximum amplitude is to be assumed. Although in absolute terms it represents smaller absorbed power values $(\bar{P} \approx 164 \mathrm{~kW})$, in the first peak $\bar{P}_{\max }$ is reached for some of the wave periods.

Fig. 2. Dimensionless time averaged absorbed power for a $1 \mathrm{~m}$ amplitude incident regular wave, assuming the power take-off mechanism simulated by a damping term and several displacement amplitude restrictions: $X_{-} Y$ means (in meters) $X=$ maximum $\left|\hat{\xi}_{1}-\hat{\xi}_{2}\right|, Y=$ maximum $\left|\hat{\xi}_{1}\right|$.

In this scenario for the power take-off mechanism the time-averaged absorbed power, given by Eq. (3), is optimized, for each wave period, as a function of the mechanical damping 
coefficient $D_{\mathrm{L}}$ (Fig. 3), the value of which should simultaneously ensure the effectiveness of the restrictions imposed on the bodies' displacement amplitudes. The highest $D_{\mathrm{L}}$ values, represented in Fig. 3, occur for wave periods corresponding to the second absorbed power peak $(T \approx 11.2 \mathrm{~s})$, although a local minimum can be observed, so that the maximum displacement amplitude for body 1 in each scenario is ensured (Fig. 4). In fact, only in this period range the damping coefficient shows a clear dependence on the maximum amplitude of the vertical displacement for body 1, presenting higher values for higher maximum amplitude values. It should be noted that, as can be observed in Fig. 3, $D_{\mathrm{L}}$ proves to be highly sensitive to wave period, so that considerably higher values are observed in a fairly narrow period band.

Fig. 3. Mechanical damping coefficient for a $1 \mathrm{~m}$ amplitude incident regular wave, assuming the power take-off mechanism simulated by a damping term and several displacement amplitude restrictions: $X \_Y$ means (in meters) $X=$ maximum $\left|\hat{\xi}_{1}-\hat{\xi}_{2}\right|, Y=$ maximum $\left|\hat{\xi}_{1}\right|$.

Fig. 4. Absolute vertical displacement amplitude for body 1 for a $1 \mathrm{~m}$ amplitude incident regular wave, assuming the power take-off mechanism simulated by a damping term and several displacement amplitude restrictions: $X_{-} Y$ means (in meters) $X=$ maximum $\left|\hat{\xi}_{1}-\hat{\xi}_{2}\right|, Y=$ maximum $\left|\hat{\xi}_{1}\right|$.

\subsection{Irregular waves}

Real sea waves are not regular but rather random, irregular, hence more suitable for a stochastic modelling. Though not allowing the planning of control strategies, the kind of modelling presented in section 2.2 should provide a more realistic overview of the device's performance in real seas, without the need to resort to the more complex time domain analysis. In the application of the stochastic model to the geometry represent in Fig. 1 the set of hydrodynamic diffraction and radiation coefficients already used for regular waves was considered. According to what has been described in section 2.2, incident waves are now represented by a frequency spectrum. In particular, a Pierson-Moskowitz formulation was adopted, expressed in terms of the sea state significant wave height $H_{\mathrm{s}}$ and energy period $T_{\mathrm{e}}$ by [13]

$$
S_{\eta}(\omega)=263 H_{\mathrm{s}}^{2} T_{\mathrm{e}}^{-4} \omega^{-5} \exp \left(-1054 T_{\mathrm{e}}^{-4} \omega^{-4}\right) .
$$

Two scenarios were considered for the power take-off equipment, namely, a first scenario in which it is simulated solely by a damping term $\left(K_{\mathrm{L}}=0\right)$ and a second in which it is simulated by both damping and spring terms. For each scenario, the values of $K_{\mathrm{L}}$ and $D_{\mathrm{L}}$ that maximize the 
averaged absorbed power (given by Eq. (19)) were computed for each sea state. Fig. 5 presents, for both scenarios, the dimensionless absorbed power, defined by $\bar{P}^{*}=\bar{P} / \bar{P}_{\max }$, in which $\bar{P}$ is here given by Eq. (19) and the maximum power extractable from a sea state, represented by the spectral distribution $S_{\eta}(\omega)$, by a heaving axisymmetric body is $\bar{P}_{\max }=\frac{g^{3} \rho}{4} \int_{0}^{\infty} \omega^{-3} S(\omega) d \omega=149.5 H_{s}^{2} T_{e}^{3}[14]$. Sea states with a $2 \mathrm{~m}$ significant wave height and wave energy period ranging from 7 to $14 \mathrm{~s}$ were considered. Generally, the device presents a better performance for sea states with smaller wave energy period. For wave periods $T_{e} \leq 10 \mathrm{~s}$ the implementation of reactive control $\left(K_{\mathrm{L}}<0-\right.$ Fig. 7) proves to be advantageous in terms of the absorbed power (Fig. 5). Nevertheless, note that in the best case presented in Fig. 5, namely for $T_{e}=7 \mathrm{~s}$ when considering a reactive power take-off, approximately only $34 \%$ of the theoretical limit $\bar{P}_{\max }$ is obtained $(\bar{P} \approx 70 \mathrm{~kW})$. This represents a significant decay comparing to what was verified in regular waves. In reality, it should be noted that the computed mechanical damping $D_{\mathrm{L}}$ (Fig. 6) and spring $K_{\mathrm{L}}$ (Fig. 7) coefficients maximize the absorbed power (Eq. (19)) for each sea state, defined by $H_{s}$ and $T_{e}$ values. Considering that, in linear theory, irregular waves are conceived as a superposition of regular waves (spectral distribution) an that the optimal coefficient $D_{\mathrm{L}}$ obtained in regular waves proved to depend strongly on the wave period $T$ (Fig. 3), the device cannot be optimally tuned simultaneously to all the frequencies in the spectrum, therefore the optimal $D_{\mathrm{L}}$ and $K_{\mathrm{L}}$ obtained for irregular waves (Figures 6 and 7 ) may be appropriate to a narrow band but not to most of the frequencies.

Fig. 5. Dimensionless time averaged absorbed power for $H_{s}=2 \mathrm{~m}$ and $T_{e}=7-14 \mathrm{~s}$, assuming the power take-off mechanism simulated by a damping term and assuming the power take-off mechanism simulated by both damping and spring terms.

Fig. 6. Mechanical damping coefficient for $H_{s}=2 \mathrm{~m}$ and $T_{e}=7-14 \mathrm{~s}$, assuming the power take-off mechanism simulated by a damping term and assuming the power take-off mechanism simulated by both damping and spring terms.

Fig. 7. Mechanical spring coefficient for $H_{s}=2 \mathrm{~m}$ and $T_{e}=7-14 \mathrm{~s}$, assuming the power take-off mechanism simulated by both damping and spring terms.

Figures 8 and 9 present the displacement variances for both body 1 and body 2, respectively for the first and second power take-off scenarios. When considering a non-reactive power take-off mechanism (first scenario) the displacement variances for the two bodies 
present similar patterns (Fig. 8). However, this is not the case when it comes to a reactive power take-off mechanism (second scenario), in which situation the patterns are significantly dissimilar (Fig. 9). In fact, the most pronounced differences between $\sigma_{\xi_{1}}^{2}$ and $\sigma_{\xi_{2}}^{2}$ occur for wave energy periods ( $T_{e} \leq 11 \mathrm{~s}$ ) to which correspond optimal mechanical spring with negative stiffness $\left(K_{\mathrm{L}}<0-\right.$ Fig. 7).

Fig. 8. Variances for the displacements of body 1 and 2 for $H_{s}=2 \mathrm{~m}$ and $T_{e}=7-14 \mathrm{~s}$, assuming the power take-off mechanism simulated by a damping term.

Fig. 9. Variances for the displacements of body 1 and 2 for $H_{s}=2 \mathrm{~m}$ and $T_{e}=7-14 \mathrm{~s}$, assuming the power take-off mechanism simulated by both damping and spring terms.

\section{Time domain results}

\section{1 $G_{m}$ parameter optimization}

The hydraulic power take-off described in section 2.3 was assumed. Masses of gas inside the high-pressure and the low-pressure accumulators $m_{1}=250 \mathrm{~kg}$ and $m_{2}=50 \mathrm{~kg}$, respectively, were considered. One single pair of cylinders was considered, with a total effective cross-sectional area $S_{c}=0.0364 \mathrm{~m}^{2}$. In each simulation initial gas pressure levels inside the accumulators were established in order to ensure temperatures $T_{i}(t)=p_{i}(t) v_{i}(t) / R$, $i=1,2$, with the gas constant (air) $R=287.0 \mathrm{~J} / \mathrm{kg} \cdot \mathrm{K}$, close to the environmental temperature $\left(T_{i}(t)=300 \mathrm{~K}\right)$.

Since the memory effect in equations (21) and (22) is negligible after a few tens of second, the infinite interval of integration in these equations may be, in practice, replaced by a finite one [9]. For the purpose of this work a 60 s interval was used. The values of the memory function defined by Eq. (23) and the limiting values of the added mass, $A_{\infty_{i j}}$, were directly obtained from WAMITC. The set of hydrodynamic diffraction and radiation damping coefficients used in the frequency domain was considered. Time series of $7200 \mathrm{~s}$, with a time step of $0.01 \mathrm{~s}$, were obtained for the several parameters that characterize the wave power device.

In the power take-off configuration, the parameter $G_{m}$ that relates the flow rate through the hydraulic motor to the pressure difference is taken as a control parameter. Fig. 10 presents the time averaged dimensionless absorbed power for irregular waves with $2 \mathrm{~m}$ significant wave height and wave energy period from 7 to $14 \mathrm{~s}$, obtained considering, for each sea state $\left(H_{s}, T_{e}\right)$, 
the optimal $G_{m}$ parameter value (which maximizes the time average of the instantaneous power available to the hydraulic motor, given by Eq. (28)) (Fig. 11). $\bar{P}^{\star}$ results are generally quite similar to the ones obtained from the stochastic model considering the first power take-off scenario (simulated by a damping term). In fact, the device performs better for sea states with smaller energy period. In particular, in both cases the highest $\bar{P}^{*}$ value is registered for sea states such that $T_{e}=7 \mathrm{~s}$ : approximately $14 \%$ of $\bar{P}_{\max }(\bar{P} \approx 29 \mathrm{~kW})$ in the hydraulic circuit's case, approximately $15 \%$ of $\bar{P}_{\max }(\bar{P} \approx 31 \mathrm{~kW})$ in the case of the stochastic model considering a non-reactive power take-off. Note that here the optimization of $G_{m}$ was not based on any preestablished algorithm but rather it consisted in an empirical process in which a fairly small number of values was tested. That is why $\bar{P}^{*}$ values in this case turned out to be slightly smaller. The optimization of $G_{m}$ corresponds to the optimization of $D_{\mathrm{L}}$ in the linear nonreactive power take-off configuration. In fact, since higher values of the parameter that relates the flow rate through the hydraulic motor to the pressure difference between the two accumulators mean smaller power take-off damping factors, it comes as no surprise that the optimal $G_{m}$ curve (Fig. 11) represents approximately the inverse image of the $D_{\mathrm{L}}$ curve in the case of a non-reactive linear power take-off (Fig. 6, black diamonds).

Fig. 10. Dimensionless time averaged absorbed power for $H_{s}=2 \mathrm{~m}$ and $T_{e}=7-14 \mathrm{~s}$, assuming a nonlinear power take-off mechanism (hydraulic circuit).

Fig. 11. Parameter that relates flow rate through the hydraulic machine to pressure difference between gas accumulators for $H_{s}=2 \mathrm{~m}$ and $T_{e}=7-14 \mathrm{~s}$.

\subsection{Phase control by latching}

The majority of the wave energy devices referred to as point absorbers present natural oscillation frequencies significantly above the range in which ocean wave energy is typically concentrated, that is $0.5<\omega<0.9 \mathrm{rad} / \mathrm{s}$ (approximately corresponding to periods $7.0<T<12.6 \mathrm{~s}$ ) [10]. In these circumstances, a mechanical spring with negative stiffness $\left(K_{\mathrm{L}}<0\right)$ should be required in order to bring the velocity of the body in phase with the diffraction force on it and thus meet the well-known optimal condition deduced for single oscillating bodies with linear power take-off mechanism in regular waves. In the case of the two-body device under study the introduction of a mechanical spring with negative stiffness also increased the absorbed power for many of the considered sea states (Fig. 7). The mechanical spring with negative stiffness, however, is a condition difficult to implement in 
reality. This can be overcome by the application of discrete phase control by latching, which consists in immobilizing the body during adequate time intervals [15]. This procedure, regarded as sub-optimal phase control by comparison with optimal reactive phase control, has been theoretically proven to be almost equally efficient for a single-body device [16]. In the context of a two-body device, by latching is meant that the bodies are constrained to remain rigidly connected to each other during convenient time intervals, so that there is no actually absolute immobilization of any of the bodies. In other words, the control strategy acts on the relative motion rather than on the absolute motions.

The use of the hydraulic circuit described in section 2.3 as power take-off introduces a natural latching mechanism, understood in the above mentioned context, in the sense that the two bodies remain rigidly connected for as long as the resulting hydrodynamic forces on its wetted surfaces do not exceed the resisting force $\Phi=S_{c} \Delta p_{a c s}$ introduced by the mechanism. In practice this means that the bodies will remain connected while $\Delta p_{c i l}<\Delta p_{a c s}$, in which $\Delta p_{\text {cil }}=p_{a}-p_{b}$ is the pressure difference in the cylinders, where $p_{a}$ is the pressure level in the upper part of the cylinders and $p_{b}$ the pressure level in the lower part. The sub-optimal method of phase control by latching here applied to the device presented in Fig. 1, proposed by Falcão (2008) [10] for a single-body device, consists in delaying the release of the bodies by forcing the rectifying valve to remain closed until the pressure difference in the cylinders equals or exceeds the pressure difference between the gas accumulators multiplied by a factor $R_{c}>1$. Hence, in this situation, the bodies remain rigidly connected while $\Delta p_{c i l}<R_{c} \Delta p_{a c s}$. The application of the method requires the optimization of $G_{m}$ together with the new parameter $R_{c}$. In fact, the value of the latching control parameter $R_{c}$ which maximizes the absorbed power does not necessarily correspond the optimal $G_{m}$ value obtained for $R_{c}=1$ [10]. The optimization of these two parameters corresponds to the optimization of $D_{\mathrm{L}}$ and $K_{\mathrm{L}}$ in the case of a linear power take-off mechanism. The use of this sub-optimal method proved to be quite effective in the case of a single-body wave energy device [10].

Fig. 12 presents the time variation of the power available to the hydraulic motor, for the interval $800 \leq t \leq 900 \mathrm{~s}$, considering an incident regular wave with $1 \mathrm{~m}$ amplitude and $9.5 \mathrm{~s}$ period, with no control (above) and with latching control assuming $R_{c}=5$ (below). In each case, the parameter $G_{m}$ is set equal to its optimal value. The application of the sub-optimal method in these terms increases by approximately $39 \%$ the time average of $P_{m}$, from $\bar{P}_{m}=157.3 \mathrm{~kW}$ in the uncontrolled scenario to $\bar{P}_{m}=218.5 \mathrm{~kW}$. It is noticeable in Fig. 12 the increment in the (less frequent) power peaks. The non-linear power take-off configuration presented in section 2.3 enables the attenuation of fluctuations in absorbed wave power, since the pressure difference between accumulators ensures the continuation of power production even when $\dot{\xi}_{2}-\dot{\xi}_{1}=0$. An immediate consequence of the application of the control strategy, visible in Fig. 12, is the 
reduction of this smoothing effect. In fact, the longer time intervals in which $\dot{\xi}_{2}-\dot{\xi}_{1}=0$, implying that the rectifying valve remains closed, represent more pronounced drops of the pressure level inside the high-pressure accumulator, which correspond to rises of the level inside the lowpressure accumulator, hence $\Delta p_{a c s}$ diminishes (Fig. 13) and so does $P_{m}$, given by Eq. (28). Note that the reduction of this smoothing effect is also connected to the larger amounts of energy absorbed from the waves as a result of the control method, since the characteristics of the hydraulic circuit were not adapted to accommodate such an increase.

Fig. 12. Power available to the hydraulic motor for a regular wave with $1 \mathrm{~m}$ amplitude and $9.5 \mathrm{~s}$ period, assuming a non-linear power take-off mechanism (hydraulic circuit), (a) with no latching control, with $G_{m}=5.0 \times 10^{-7} \mathrm{~m} / \mathrm{Ns}$, and (b) with latching control, with $G_{m}=1.5 \times 10^{-6} \mathrm{~m} / \mathrm{Ns}$ and $R_{c}=5$.

Fig. 13. Pressure inside the high-pressure gas accumulator $\left(p_{1}\right)$, inside the low-pressure accumulator $\left(p_{2}\right)$ and pressure difference between the accumulators $\left(\Delta p_{a c s}\right)$ for a regular wave with $1 \mathrm{~m}$ amplitude and $9.5 \mathrm{~s}$ period, assuming a non-linear power take-off mechanism (hydraulic circuit), (a) with no latching control, with $G_{m}=5.0 \times 10^{-7} \mathrm{~m} / \mathrm{Ns}$, and (b) with latching control, with $G_{m}=1.5 \times 10^{-6} \mathrm{~m} / \mathrm{Ns}$ and $R_{c}=5$.

For irregular wave conditions, the improvements in the device's performance resulting from the application of the sub-optimal phase control by latching were not so evident. In the best tested scenario, for $H_{s}=2 \mathrm{~m}$ and $T_{e}=7 \mathrm{~s}$, the value of the time average absorbed power was increased by approximately $9 \%$, when compared to the uncontrolled scenario. Fig. 14 presents the time variation of the relative velocity between the two bodies, for the interval $2200 \leq t \leq 2400 \mathrm{~s}$, with no control $\left(R_{c}=1\right)$, considering $G_{m}=1.0 \times 10^{-6} \mathrm{~m} / \mathrm{Ns}$ (black line), and with latching control, considering $G_{m}=2.0 \times 10^{-6} \mathrm{~m} / \mathrm{Ns}$ and $R_{c}=5$ (red dashed line). One can observe, even in the uncontrolled scenario (Fig. 14, black line), the occurrence of time intervals in which there is no relative displacement between the bodies, as a consequence of the resulting hydrodynamic forces on the bodies' wetted surfaces not exceeding the resisting force introduced by the power take-off mechanism. These time intervals appear significantly stretched in the controlled scenario (Fig. 14, red dashed line). As a consequence, the less frequent relative velocity peaks appear considerably more accentuated, i.e., the application of the control strategy considerably increases $\left|\dot{\xi}_{2}-\dot{\xi}_{1}\right|$. In fact, if higher $\left|\dot{\xi}_{2}-\dot{\xi}_{1}\right|$ peaks mean higher $P_{m}$ peaks (Fig. 15), longer periods in which $\dot{\xi}_{2}-\dot{\xi}_{1}=0$ also mean longer and more pronounced $P_{m}$ decays, so that power fluctuations appear less smoothened in the controlled scenario. 
Fig. 14. Relative velocity between the two bodies for $H_{s}=2 \mathrm{~m}$ and $T_{e}=7 \mathrm{~s}$, assuming a non-linear power take-off mechanism (hydraulic circuit), with no latching control $\left(R_{c}=1\right)$, considering $G_{m}=1.0 \times 10^{-6} \mathrm{~m} / \mathrm{Ns}$, and with latching control, considering $G_{m}=2.0 \times 10^{-6} \mathrm{~m} / \mathrm{Ns}$ and $R_{c}=5$.

Fig. 15. Power available to the hydraulic motor for $H_{s}=2 \mathrm{~m}$ and $T_{e}=7 \mathrm{~s}$, assuming a non-linear power take-off mechanism (hydraulic circuit), with no latching control $\left(R_{c}=1\right)$, considering $G_{m}=1.0 \times 10^{-6} \mathrm{~m} / \mathrm{Ns}$, and with latching control, considering $G_{m}=2.0 \times 10^{-6} \mathrm{~m} / \mathrm{Ns}$ and $R_{c}=5$.

Though it is known that optimal phase control in irregular waves requires the prediction of the incoming wave field [17], the time-averaged absorbed power increments here verified, resulting from the application of the sub-optimal phase control by latching method, are considerably less significant than the ones reported in [10] in the case of a single-body device (by a factor about 2.3-2.8). This evidence is also valid for regular waves ([10] reports increments by a factor up to about 3.8). The smaller effectiveness of the method in this case should be related to the fact that the strategy does not act upon the absolute displacement of the bodies, rather it imposes only constraints on the relative displacement.

\section{Pontryagin Maximum Principle}

The maximum principle of Pontryagin (PMP) [11] was first applied to wave energy converter control problems in [18] and [19]. We applied PMP to improve the performance of the device, particularly in irregular waves. Since similar performances were found to be attainable with a linear damper and with a non-linear hydraulic circuit, this led us to simulate the power take-off by a damping term. In these circumstances, it is assumed that the load has the form

$$
f_{\mathrm{L}}\left(\dot{\xi}_{i}\right)= \pm D_{\mathrm{L}}(t)\left(\dot{\xi}_{1}(t)-\dot{\xi}_{2}(t)\right)
$$

The PMP aims to find the optimal control variable $D_{\mathrm{L}}^{*}(t)$ which maximizes the power production for the time interval $T$

$$
\bar{P}=\frac{1}{T} \int_{0}^{T} D_{\mathrm{L}}^{*}(t)\left(\dot{\xi}_{1}(t)-\dot{\xi}_{2}(t)\right)^{2} d t
$$

Taking into consideration that $k_{i j}(t)=\frac{d L_{i j}(t)}{d t}$, the convolution integrals presented in equations (21) and (22) may be written as 
$\int_{-\infty}^{t} L_{i j}(t-\tau) \frac{d \dot{\xi}_{j}(\tau)}{d \tau} d \tau=\int_{-\infty}^{t} k_{i j}(t-\tau) \dot{\xi}_{j}(\tau) d \tau$.

The state equations (21) and (22) may now be given by

$\frac{d \dot{\xi}_{1}}{d t}=\frac{1}{A_{\infty 12}^{2}-\left(M_{1}+A_{\infty_{11}}\right)\left(M_{2}+A_{\infty 22}\right)} \times\left(\begin{array}{l}\left(M_{2}+A_{\infty 22}\right)\left(\begin{array}{l}\int_{-\infty}^{t} k_{11}(t-\tau) \dot{\xi}_{1}(\tau) d \tau+C_{1} \xi_{1}(t)+ \\ \left.\int_{-\infty}^{t} k_{12}(t-\tau) \dot{\xi}_{2}(\tau) d \tau-f_{D_{1}}(t)+D_{\mathrm{L}}(t)\left(\dot{\xi}_{1}(t)-\dot{\xi}_{2}(t)\right)\right)\end{array}\right)- \\ -A_{\infty_{12}}\left(\begin{array}{l}C_{2} \xi_{2}(t)-t_{\mathrm{D}_{2}}(t)+\int_{-\infty}^{t} k_{21}(t-\tau) \dot{\xi}_{1}(\tau) d \tau+ \\ +\int_{-\infty}^{t} k_{22}(t-\tau) \dot{\xi}_{2}(\tau) d \tau-D_{\mathrm{L}}(t)\left(\dot{\xi}_{1}(t)-\dot{\xi}_{2}(t)\right)\end{array}\right)\end{array}\right)$

$\frac{d \dot{\xi}_{2}}{d t}=\frac{1}{A_{\infty_{12}}^{2}-\left(M_{1}+A_{\infty_{11}}\right)\left(M_{2}+A_{\infty_{22}}\right)} \times\left(\begin{array}{l}\left(M_{1}+A_{\infty_{11}}\left(\begin{array}{l}\int_{-\infty}^{t} k_{21}(t-\tau) \dot{\xi}_{1}(\tau) d \tau+C_{2} \xi_{2}(t)+ \\ \left.\int_{-\infty}^{t} k_{22}(t-\tau) \dot{\xi}_{2}(\tau) d \tau-f_{\mathrm{D}_{2}}(t)-D_{\mathrm{L}}(t)\left(\dot{\xi}_{1}(t)-\dot{\xi}_{2}(t)\right)\right)\end{array}\right)-\left(\begin{array}{l}C_{1} \xi_{1}(t)-f_{\mathrm{D}_{1}}(t)+\int_{-\infty}^{t} k_{11}(t-\tau) \dot{\xi}_{1}(\tau) d \tau+ \\ +A_{\infty_{12}}^{t} k_{12}(t-\tau) \dot{\xi}_{2}(\tau) d \tau+D_{\mathrm{L}}(t)\left(\dot{\xi}_{1}(t)-\dot{\xi}_{2}(t)\right)\end{array}\right)\right.\end{array}\right)$

$\frac{d \xi_{1}}{d t}=\dot{\xi}_{1}$

$\frac{d \xi_{2}}{d t}=\dot{\xi}_{2}$

Thus, the state variables in these equations are $\xi_{1}, \xi_{2}, \dot{\xi}_{1}$ and $\dot{\xi}_{2}$.

The Hamiltonian is defined in terms of the state equations, adjoint variables $\lambda_{i}$ and the function to be maximized (31), so that

$$
H=D_{\mathrm{L}}(t)\left(\dot{\xi}_{1}(t)-\dot{\xi}_{2}(t)\right)^{2}+\lambda_{1} \frac{d \xi_{1}}{d t}+\lambda_{2} \frac{d \xi_{2}}{d t}+\lambda_{3} \frac{d \dot{\xi}_{1}}{d t}+\lambda_{4} \frac{d \dot{\xi}_{2}}{d t} .
$$

The adjoint variables $\lambda_{i}$ are obtained from adjoint equations which, according to PMP, are defined in terms of the Hamiltonian by

$$
\frac{d \lambda_{1}}{d t}=-\frac{\partial H}{\partial \xi_{1}}, \frac{d \lambda_{2}}{d t}=-\frac{\partial H}{\partial \xi_{2}}, \frac{d \lambda_{3}}{d t}=-\frac{\partial H}{\partial \dot{\xi}_{1}}, \frac{d \lambda_{4}}{d t}=-\frac{\partial H}{\partial \dot{\xi}_{2}} .
$$


Since $H=H\left(D_{\mathrm{L}}, \xi_{1}, \xi_{2}, \dot{\xi}_{1}, \dot{\xi}_{2}, \lambda_{1}, \lambda_{2}, \lambda_{3}, \lambda_{4}\right)$, following the PMP it should be for the optimal control variable

$$
H\left(D_{\mathrm{L}}^{*}, \xi_{1}^{*}, \xi_{2}^{*}, \dot{\xi}_{1}^{*}, \dot{\xi}_{2}^{*}, \lambda_{1}, \lambda_{2}, \lambda_{3}, \lambda_{4}\right)=\operatorname{Max}_{D_{\mathrm{L}} \in \Omega} H\left(D_{\mathrm{L}}, \xi_{1}^{*}, \xi_{2}^{*}, \dot{\xi}_{1}^{*}, \dot{\xi}_{2}^{*}, \lambda_{1}, \lambda_{2}, \lambda_{3}, \lambda_{4}\right) \quad \forall t \in T .
$$

Here * denotes the optimal solution and $\Omega$ is the admissible domain for the control variable $D_{\mathrm{L}}$. It follows from equations (33)-(37) that the Hamiltonian is linear for the control variable. Therefore $D_{\mathrm{L}}$ should take the minimum or maximum values of $\Omega$ except for singular arcs. In particular,

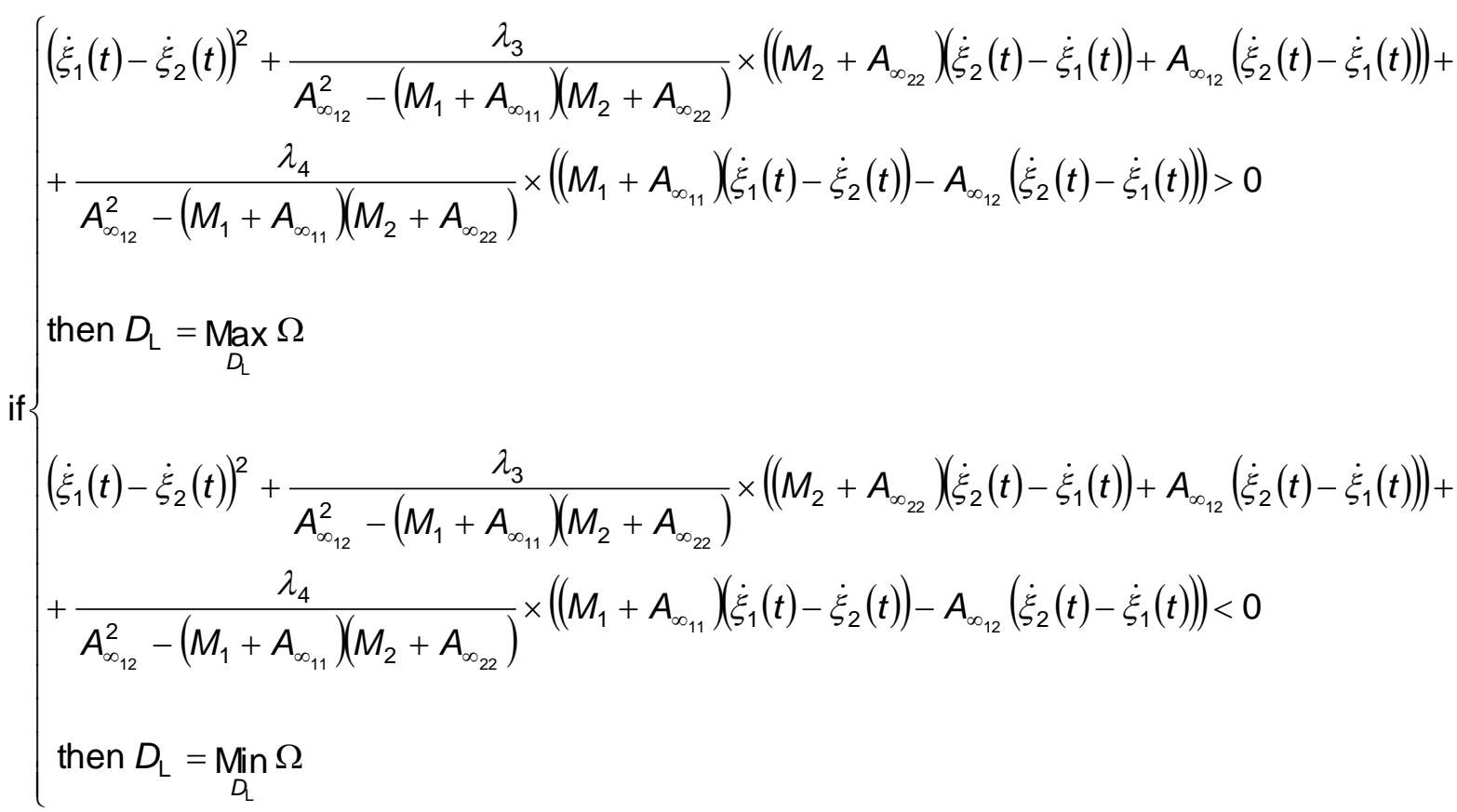

For the time intervals in which

$$
\begin{aligned}
& \left(\dot{\xi}_{1}(t)-\dot{\xi}_{2}(t)\right)^{2}+\frac{\lambda_{3}}{A_{\infty_{12}}^{2}-\left(M_{1}+A_{\infty_{11}}\right)\left(M_{2}+A_{\infty_{22}}\right)} \times\left(\left(M_{2}+A_{\infty_{22}}\right)\left(\dot{\xi}_{2}(t)-\dot{\xi}_{1}(t)\right)+A_{\infty_{12}}\left(\dot{\xi}_{2}(t)-\dot{\xi}_{1}(t)\right)\right)+ \\
& +\frac{\lambda_{4}}{A_{\infty_{12}}^{2}-\left(M_{1}+A_{\infty_{11}}\right)\left(M_{2}+A_{\infty_{22}}\right)} \times\left(\left(M_{1}+A_{\infty_{11}}\right)\left(\dot{\xi}_{1}(t)-\dot{\xi}_{2}(t)\right)-A_{\infty_{12}}\left(\dot{\xi}_{2}(t)-\dot{\xi}_{1}(t)\right)\right)=0,
\end{aligned}
$$

singular arcs will be present in the solution and the control variable may take values between the minimum and maximum values of $\Omega$. The value to be considered for the control variable will be implicitly given by the conditional gradient algorithm used to solve the problem [20].

The verification of the conditions (40) implies the computation of the adjoint variables for the time interval $T$. Applying the transversality equations and assuming that the initial values for the state variables are known but not $\xi_{1}(T), \xi_{2}(T), \dot{\xi}_{1}(T)$ and $\dot{\xi}_{2}(T)$, it will be $\lambda_{1}(T)=\lambda_{2}(T)=\lambda_{3}(T)=\lambda_{4}(T)=0$. 
The application of the PMP implies solving equations (38) in the reverse order in time, meaning that it represents a non-causal problem in which the previous knowledge of the diffraction forces applied on the device during the time interval $T$ is needed. Hence, based on the PMP, a control strategy considered to be causal was established. Since PMP states that the optimal load damping coefficient should take the $\Omega$ minimum or maximum values (except for singular arcs), it is assumed that $D_{\mathrm{L}}=\left\{\operatorname{Min}_{D_{\mathrm{L}}} \Omega, \operatorname{Max}_{D_{\mathrm{L}}} \Omega\right\}$ for most of the time interval $T$. The adopted procedure consists in setting inferior and superior relative velocity limits, $\left|\dot{\xi}_{2}(t)-\dot{\xi}_{1}(t)\right|_{\text {inf }}$ and $\left|\dot{\xi}_{2}(t)-\dot{\xi}_{1}(t)\right|_{\text {sup }}$, below and above which $D_{\mathrm{L}}$ should take the value $\underset{D_{\mathrm{L}}}{\operatorname{Min} \Omega}$ and $\operatorname{Max}_{D_{\mathrm{L}}} \Omega$, respectively. In the intermediate region $D_{\mathrm{L}}$ takes a value given by a linear regression. Note that this strategy will introduce large fluctuations in the power production, characteristic of power take-off mechanism configurations with no energy storage system. This may damage the energy quality from the electrical grid point of view (this problem might be mitigated by setting several devices in large array configurations). It should also be stressed out that this strategy is not an optimum control method but instead it is based on the findings of PMP.

Table 1 presents the time averaged absorbed power for $1 \mathrm{~m}$ amplitude incident regular waves with periods $7.5 \mathrm{~s}$ and $8.5 \mathrm{~s}$, for both the uncontrolled scenario and the best controlled scenarios. For $T=7.5 \mathrm{~s}$ a $35 \%$ absorbed power increase is observed. For $T=8.5 \mathrm{~s}$ the increase merely approaches $21 \%$. Fig. 16 shows, for $T=7.5 \mathrm{~s}$, the time series for the relative velocity between the two bodies and the mechanical damping coefficient according to the established algorithm. As expected, this leads to larger time-averaged values of $P$ (Fig. 17).

Table 1. Time averaged absorbed power assuming a power take-off mechanism simulated by a damping term uncontrolled and controlled (strategy based on PMP), for $1 \mathrm{~m}$ amplitude incident regular waves with $7.5 \mathrm{~s}$ and $8.5 \mathrm{~s}$ periods.

Fig. 16. Relative velocity between the two bodies and mechanical damping coefficient for a regular wave with $1 \mathrm{~m}$ amplitude and $7.5 \mathrm{~s}$ period, considering $\left|\dot{\xi}_{2}(t)-\dot{\xi}_{1}(t)\right|_{\text {inf }}=0.55 \mathrm{~m} / \mathrm{s},\left|\dot{\xi}_{2}(t)-\dot{\xi}_{1}(t)\right|_{\text {sup }}=0.6 \mathrm{~m} / \mathrm{s}$, $\operatorname{Min} \Omega=3.9 \mathrm{kNs} / \mathrm{m}$ and $\operatorname{Max} \Omega=586.0 \mathrm{kNs} / \mathrm{m}$.

Fig. 17. Absorbed power for a regular wave with $1 \mathrm{~m}$ amplitude and 7.5s period, for the uncontrolled scenario and for the controlled scenario considering $\left|\dot{\xi}_{2}(t)-\dot{\xi}_{1}(t)\right|_{\text {inf }}=0.55 \mathrm{~m} / \mathrm{s},\left|\dot{\xi}_{2}(t)-\dot{\xi}_{1}(t)\right|_{\text {sup }}=0.6 \mathrm{~m} / \mathrm{s}$, $\underset{D}{\operatorname{Min}} \Omega=3.9 \mathrm{kNs} / \mathrm{m}$ and $\underset{D_{L}}{\max } \Omega=586.0 \mathrm{kNs} / \mathrm{m}$. 
In irregular waves conditions the benefits from the control strategy are not so clear. Table 2 presents the results for $H_{s}=2 \mathrm{~m}, T_{e}=9 \mathrm{~s}$ and $T_{e}=10 \mathrm{~s}$. For both sea states a $4 \% \bar{P}$ increase is observable. Observing the time variation of $\dot{\xi}_{2}-\dot{\xi}_{1}$ and $D_{\mathrm{L}}$ for $T_{e}=10 \mathrm{~s}$ (Fig. 18), we may notice the occurrence of time intervals in which the relative velocity does not reach $\left|\dot{\xi}_{2}(t)-\dot{\xi}_{1}(t)\right|_{\text {sup }}=0.2 \mathrm{~m} / \mathrm{s}$, so that $D_{\mathrm{L}}$ does not take the maximum value $\operatorname{Max}_{D_{\mathrm{L}}} \Omega=4580.3 \mathrm{kNs} / \mathrm{m}$. Nevertheless, it is generally in the time intervals in which $D_{\mathrm{L}}$ saturates in the maximum value that the absorbed power peaks in the controlled scenario are less accentuated than the ones in the uncontrolled scenario (Fig. 19).

Table 2. Time averaged absorbed power assuming a power take-off mechanism simulated by a damping term uncontrolled and controlled (strategy based on PMP), for $H_{s}=2 \mathrm{~m}, T_{e}=9 \mathrm{~s}$ and $T_{e}=10 \mathrm{~s}$.

Fig. 18. Relative velocity between the two bodies and mechanical damping coefficient for $H_{s}=2 \mathrm{~m}$ and $T_{e}=10 \mathrm{~s}$, considering $\left|\dot{\xi}_{2}(t)-\dot{\xi}_{1}(t)\right|_{\text {inf }}=0.15 \mathrm{~m} / \mathrm{s},\left|\dot{\xi}_{2}(t)-\dot{\xi}_{1}(t)\right|_{\text {sup }}=0.2 \mathrm{~m} / \mathrm{s}, \underset{D_{L}}{\operatorname{Min}} \Omega=30.5 \mathrm{kNs} / \mathrm{m}$ and $\operatorname{Max}_{D_{\mathrm{L}}} \Omega=4580.3 \mathrm{kNs} / \mathrm{m}$.

Fig. 19. Absorbed power for $H_{s}=2 \mathrm{~m}$ and $T_{e}=10 \mathrm{~s}$, for the uncontrolled scenario and for the controlled scenario considering $\left|\dot{\xi}_{2}(t)-\dot{\xi}_{1}(t)\right|_{\text {inf }}=0.15 \mathrm{~m} / \mathrm{s}, \quad\left|\dot{\xi}_{2}(t)-\dot{\xi}_{1}(t)\right|_{\text {sup }}=0.2 \mathrm{~m} / \mathrm{s}, \quad \operatorname{Min}_{D_{\mathrm{L}}} \Omega=30.5 \mathrm{kNs} / \mathrm{m}$ and $\operatorname{Max}_{D_{L}} \Omega=4580.3 \mathrm{kNs} / \mathrm{m}$

In opposition to what is observed in regular waves, it seems clear that the characteristics of irregular waves difficult the setting of control conditions to fit the totality of the time series. In reality, it is not viable, in these conditions, to simultaneously tune the device to all the spectrum components, since a control parameter should be only adequate to a restricted frequency range.

\section{Conclusions}

Frequency-domain (regular waves), stochastic and time-domain models were developed for a device composed by two coaxial axisymmetric bodies. Frequency-domain modelling for regular waves allows tuning of the device to the frequencies representative of the typical wave 
spectrum for the intended deployment site. In the absence of restrictions, reactive control allows the device to extract the theoretical maximum power. Mechanical damping coefficient proved to be highly sensitive to wave period. As a consequence, the device's performance is significantly poorer in irregular waves, as it was verified by means of stochastic modelling for irregular waves.

The use of the stochastic model allows finding variances that define Gaussian probability density functions for relevant wave device parameters. It was assumed that the power take-off mechanical equipment has a linear behaviour and can be modelled by spring and damping coefficients. Its characteristics were assumed to be constant for the duration of a sea state. Reactive control proved to increase absorbed power only for sea states with energy period smaller than 10s. The stochastic model produces accurate results in irregular waves, so that it can be a useful tool in the preliminary assessment of the devices' performance in more realistic sea conditions.

For the time-domain model a non-linear power take-off mechanism configuration was devised, consisting on a hydraulic circuit with low-pressure and high-pressure gas accumulators. Results for irregular waves showed a good agreement with the ones obtained from the stochastic model considering a linear damper. The results showed that the optimization of the parameter that relates flow rate through the hydraulic machine to pressure difference between the two accumulators corresponds to the optimization of the damping coefficient in the case of the stochastic model with a non-reactive linear power take-off mechanism.

In order to improve the device's performance, the sub-optimal method of phase control by latching proposed by [10] was tested. This method proved to be effective for a single-body device. This, however, was not the case for the two-body device, in particular in irregular waves conditions. In fact, the method, in this context, does not act on the absolute displacement of the bodies. It imposes constraints only on the relative displacement.

A control strategy based on the analytically development of Pontryagin Maximum Principle was established, considering the power take-off mechanism simulated by a damping term. The strategy was empirically tested for incident regular and irregular waves. For regular waves a considerable absorbed power increase was registered. This, however, was not the case for irregular waves, in which situation marginal increases were registered.

Further control strategies should be required in order to improve the performance of twobody devices in real waves conditions. It should be taken into consideration that it is not possible to simultaneously tune the device to all the spectrum frequencies. The development of control strategies appropriate to implementation in real time is essential. 


\section{Acknowledgment}

The work here reported was supported by the Portuguese Foundation for Science and Technology under the contract PTDC/EME-MFE/66999/2006.

\section{References}

[1] Falnes J. Wave-energy conversion through relative motion between two single-mode oscillating bodies. Journal of Offshore Mechanics and Arctic Engineering 1999;121:32-38.

[2] Srokosz MA, Evans DV. A theory for wave-power absorption by two independently oscillating bodies. Journal of Fluid Mechanics 1979;90(2):337-62.

[3] Korde, UA. Systems of reactively loaded coupled oscillating bodies in wave energy conversion. Applied Ocean Research 2003;25:79-91.

[4] Mavrakos, SA. Hydrodynamic coefficients in heave of two concentric surface-piercing truncated circular cylinders. Applied Ocean Research 2004;26:84-97.

[5] Ó'Catháin M, Leira JBJ, Ringwood JV, Gilloteaux JC. A modelling methodology for multibody systems with application to wave-energy devices. Ocean Engineering 2008;35(13):1381-87.

[6] Clément A, Babarit A, Gilloteaux JC, Josset C, Duclos G. The SEAREV wave energy converter. In: Proceedings of the $6^{\text {th }}$ European Wave and Tidal Energy Conference, Glasgow, UK; 2005.

[7] Justino PAP, Clément A. Hydrodynamic performance for small arrays of submerged spheres. In: Proceedings of the $5^{\text {th }}$ European Wave Energy Conference, Cork, Ireland; 2003.

[8] Falcão AF de O, Rodrigues R. Stochastic modelling of OWC wave power plant performance. Applied Ocean Research 2002;24:59-71.

[9] Falcão AF de O. Modeling and control of oscillating-body wave energy converters with hydraulic power take-off and gas accumulator. Ocean Engineering 2007;34:2021-32.

[10] Falcão AF de O. Phase control through load control of oscillating-body wave energy converters with hydraulic PTO system. Ocean Engineering 2008;34:358-66.

[11] Pontryagin LS, Boltyanskii VG, Gamkrelidze RV, Mishchenko EF. The Mathematical Theory of Optimal Processes. New York, USA: John Wiley; 1962. 
[12] Falnes J. Ocean waves and oscillating systems. Cambridge, UK: Cambridge University Press; 2002.

[13] Goda Y. Random Seas and Design of Marine Structures. Tokyo, Japan: University of Tokyo Press; 1985.

[14] Falcão AF de O, Justino PAP, Henriques JCC, André JMCS. Modelling and control of the IPS wave power buoy. In: Proceedings of the $2^{\text {nd }}$ International Conference on Ocean Energy, Brest, France; 2008.

[15] Budal K, Falnes J. Interacting point absorbers with controlled motion, Power from Sea Waves. ed. B Count. London, UK: Academic Press; 1980. p. 381-99.

[16] Hals J, Bjarte-Larsson T, Falnes J. Optimum reactive control and control by latching of a wave-absorbing semisubmerged heaving sphere. In: Proceedings of the $21^{\text {st }}$ International Conference on Offshore Mechanics and Arctic Engineering, Oslo, Norway; 2002. Article no. 28172 available on CD. ISBN 0-7918-3599-5.

[17] Falnes J. Optimum control of oscillation of wave-energy converters. International Journal of Offshore and Polar Engineering 2002;12(2):147-155.

[18] Hoskin RE, Nichols NK. Optimal strategies for phase control of wave energy devices. In: Utilization of ocean waves: wave to energy conversion. 345 East 47th Street New York, New York 10017-2398: American Society of Civil Engineers; 1986. p. 184-99.

[19] Hoskin RE, Count BM, Nichols NK, Nichol DAC. Phase control for the oscillating water column. In: Evans DV, Falcão AF de O (Eds.). Hydrodynamics of Ocean Wave-Energy Utilization. Berlin, Germany: Springer; 1986. p. 257-268.

[20] Birkett NRC, Nichols NK. Optimal control problems in tidal power generation. Department of Mathematics, University of Reading; 1983. Rpt. NA8/83. 


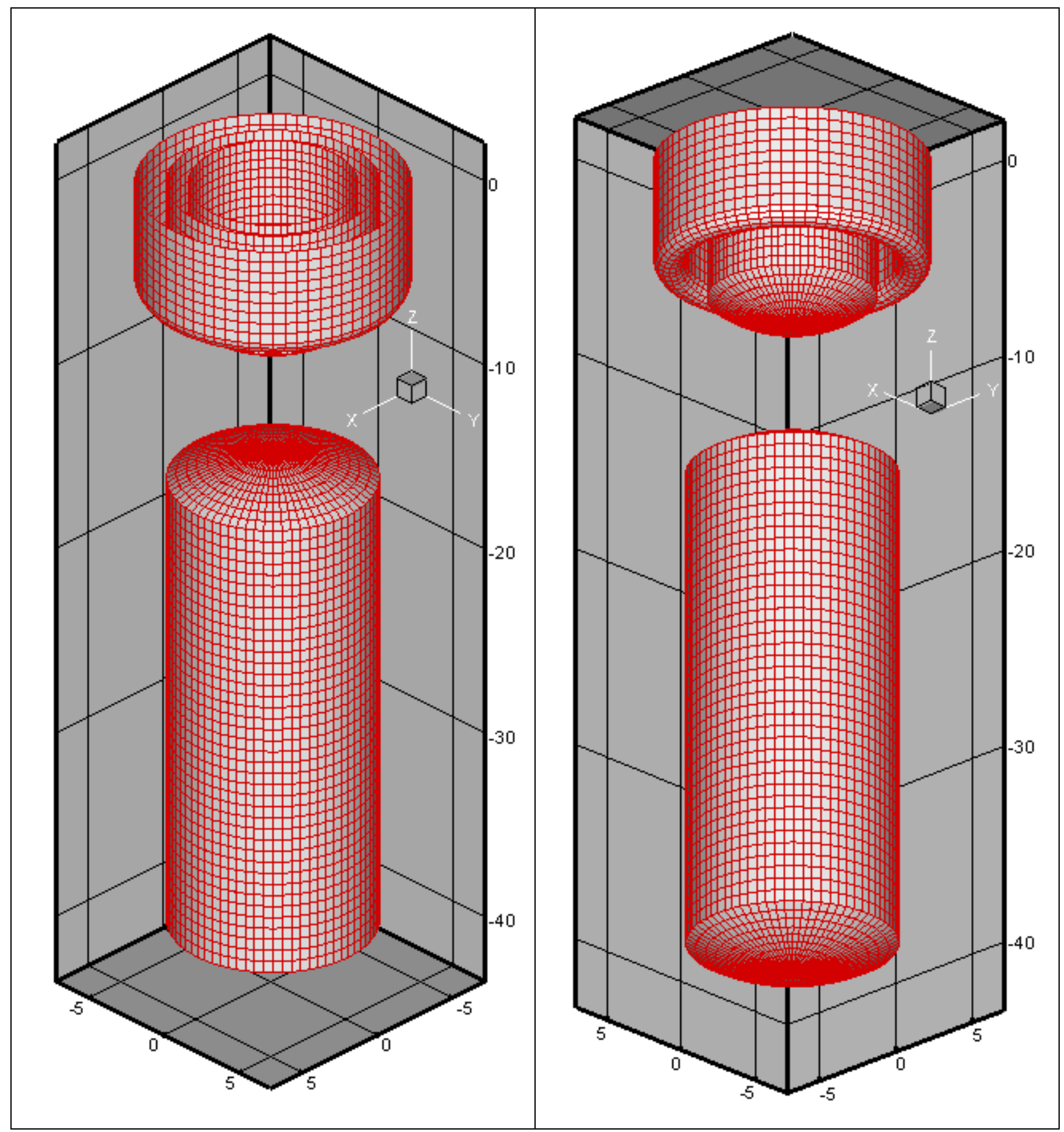




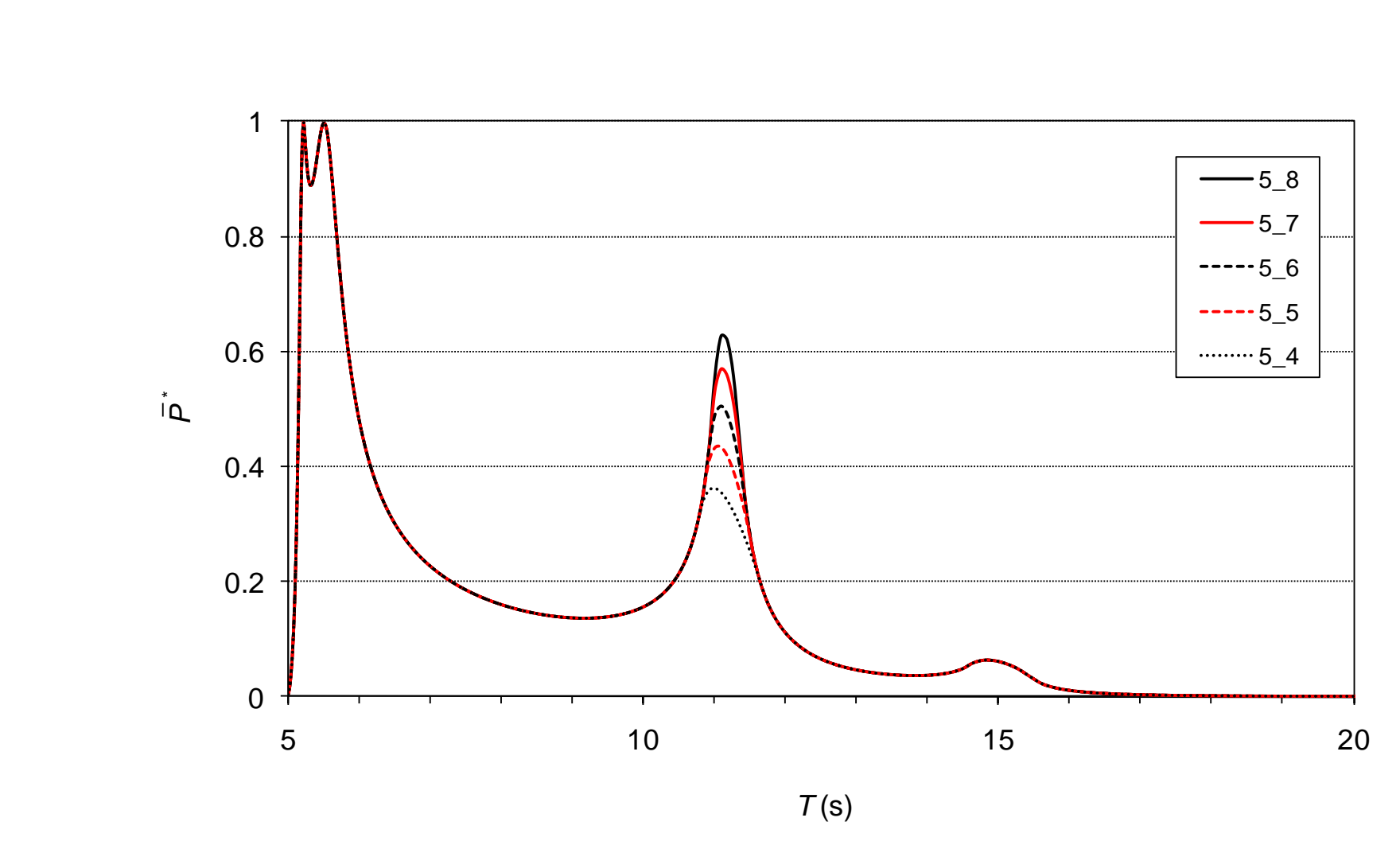

Figure2

Figure2

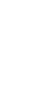

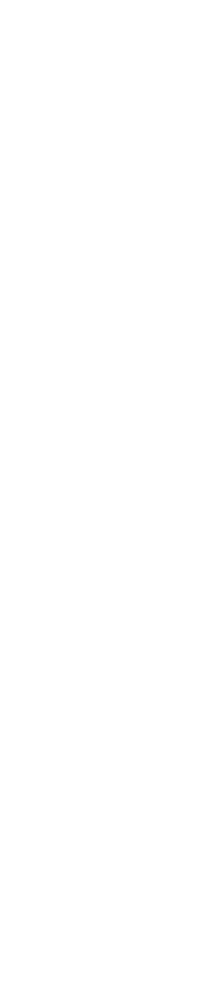

(
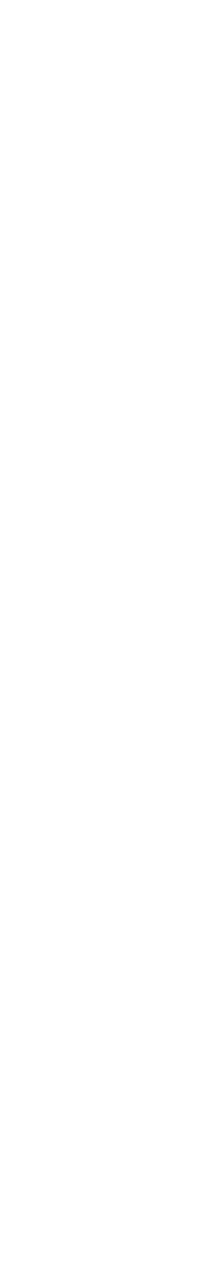

.

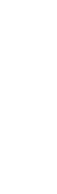

. 


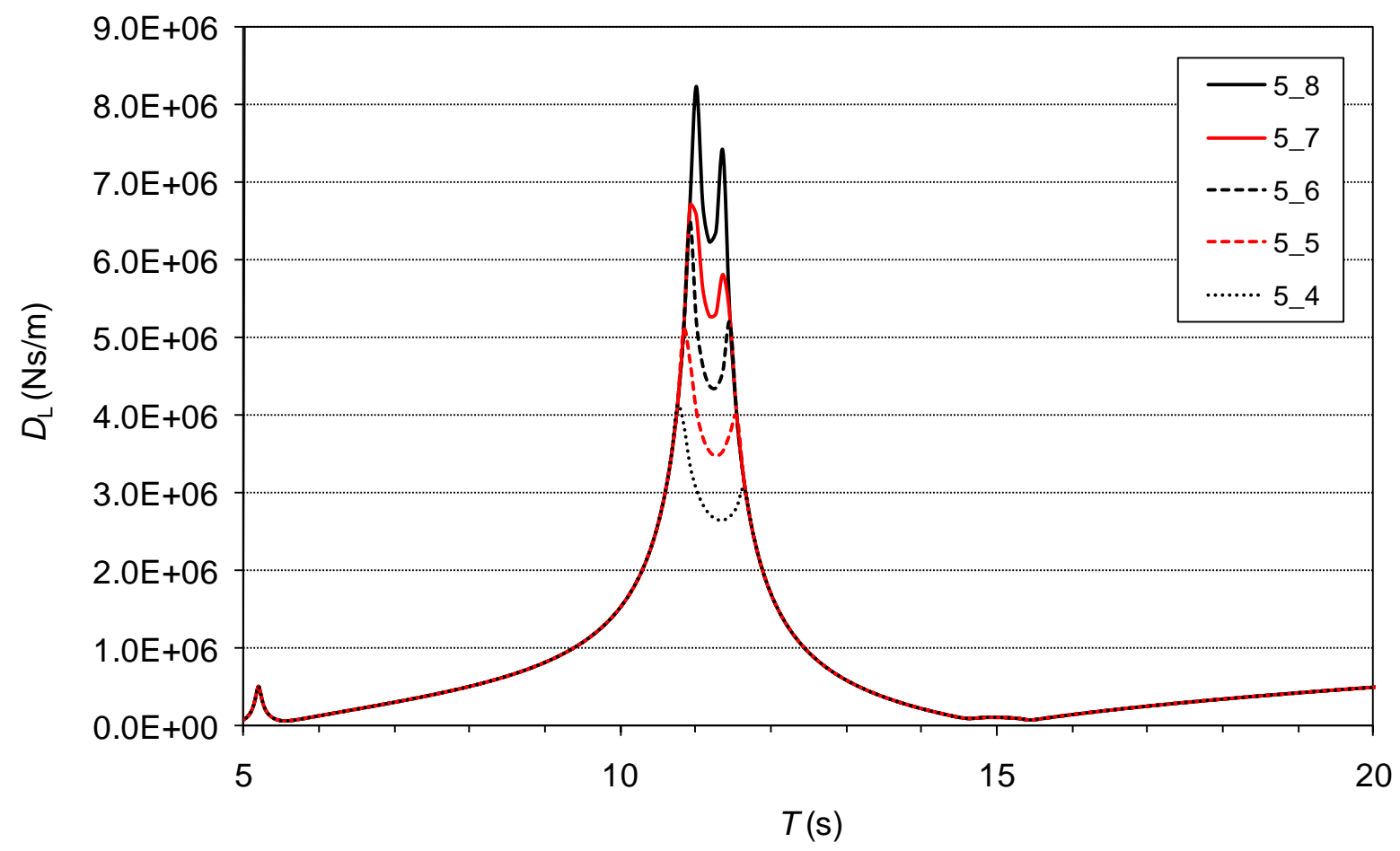




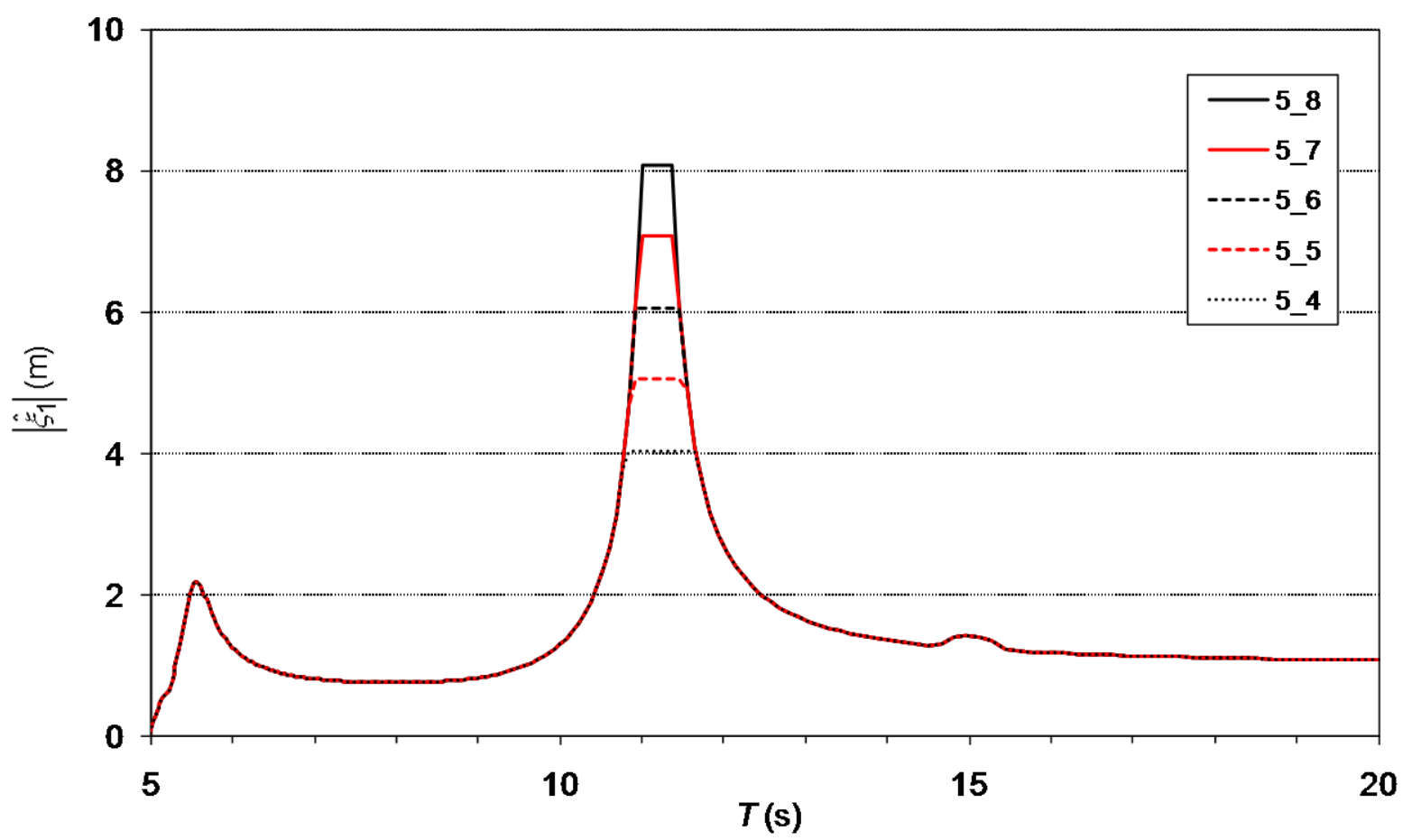




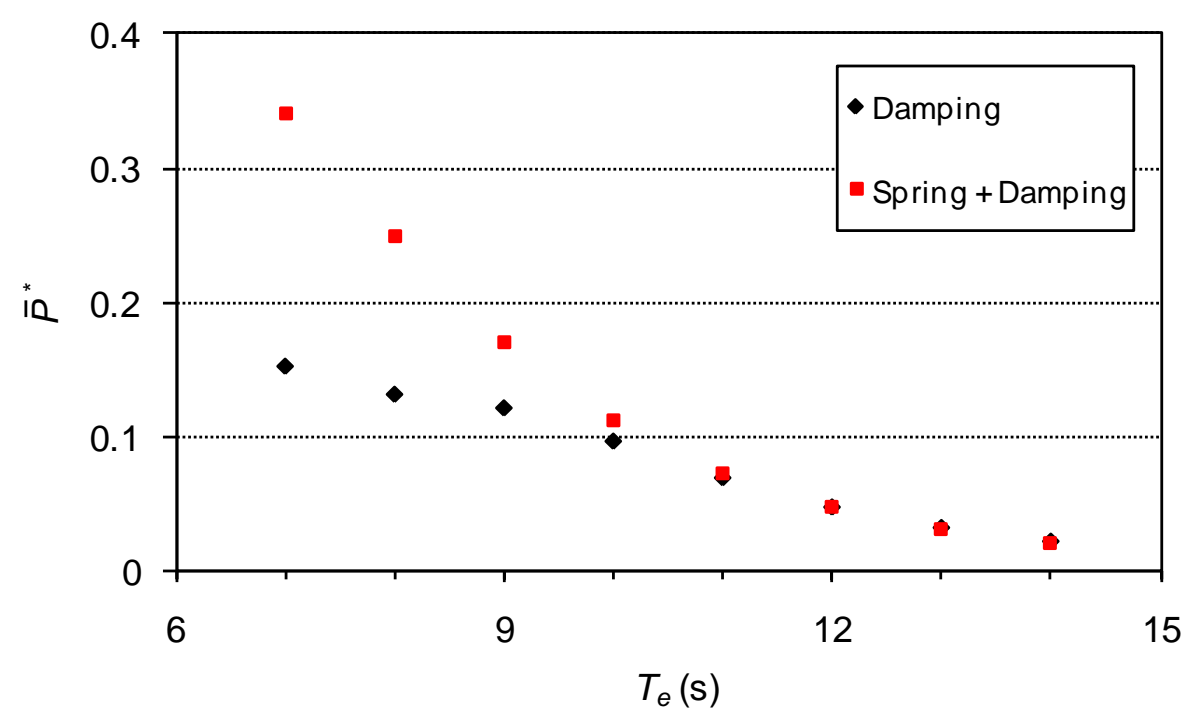




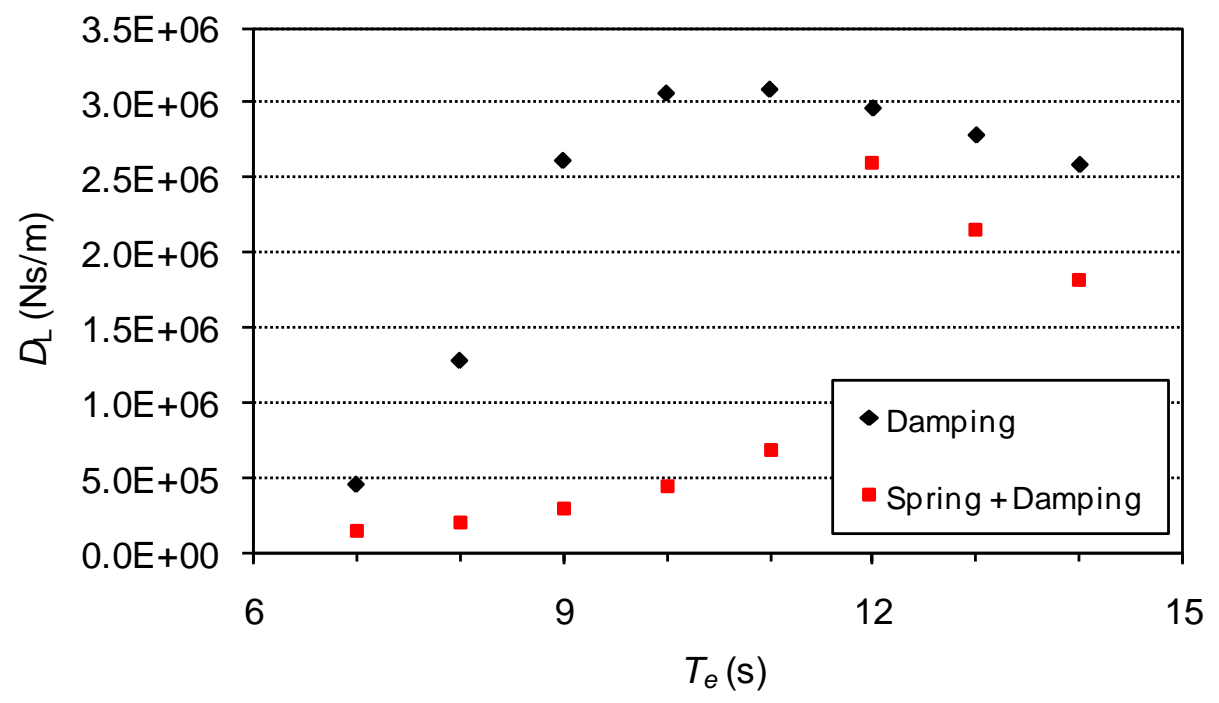




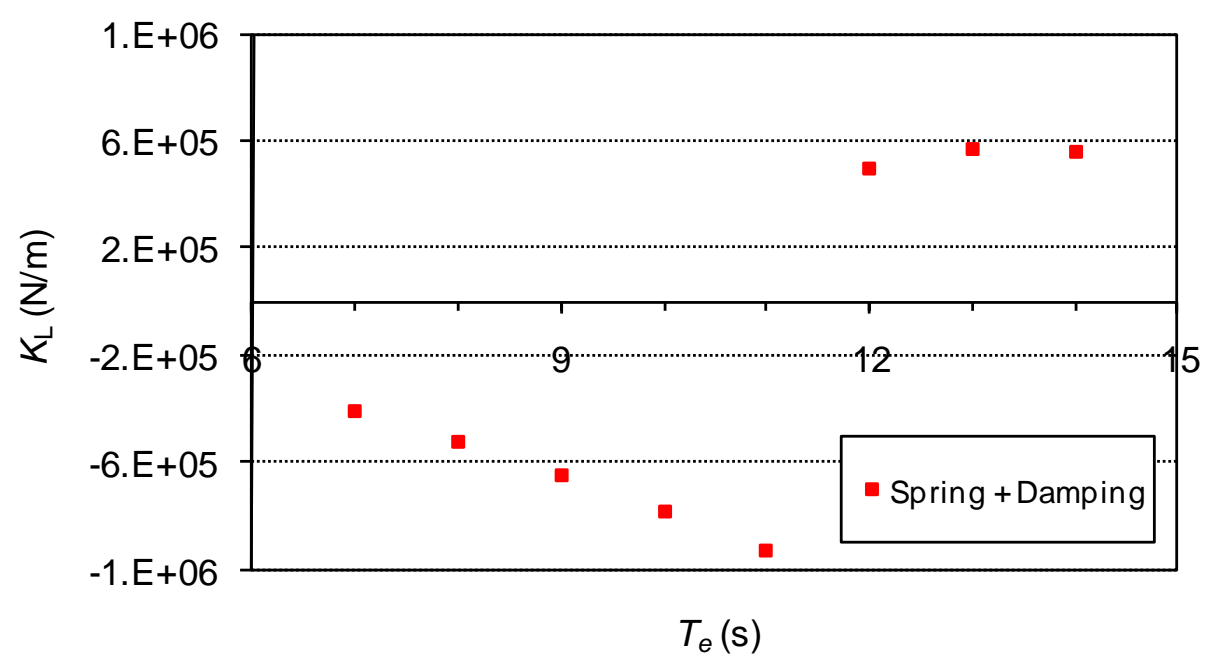




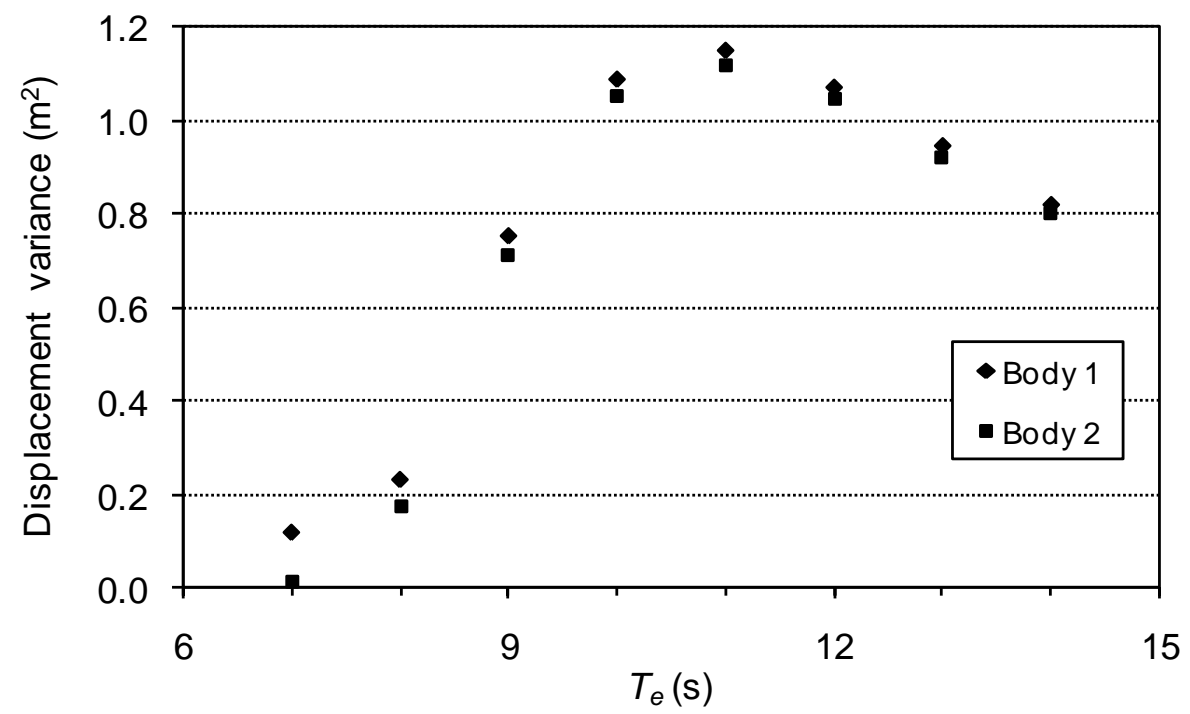




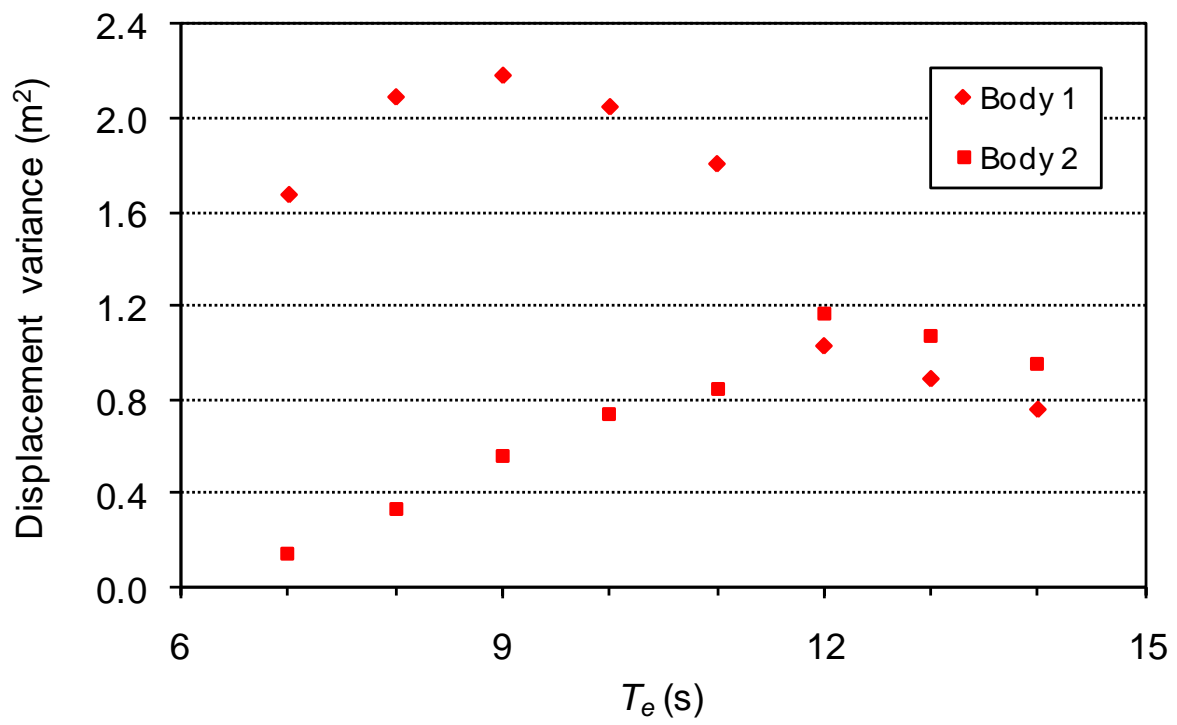




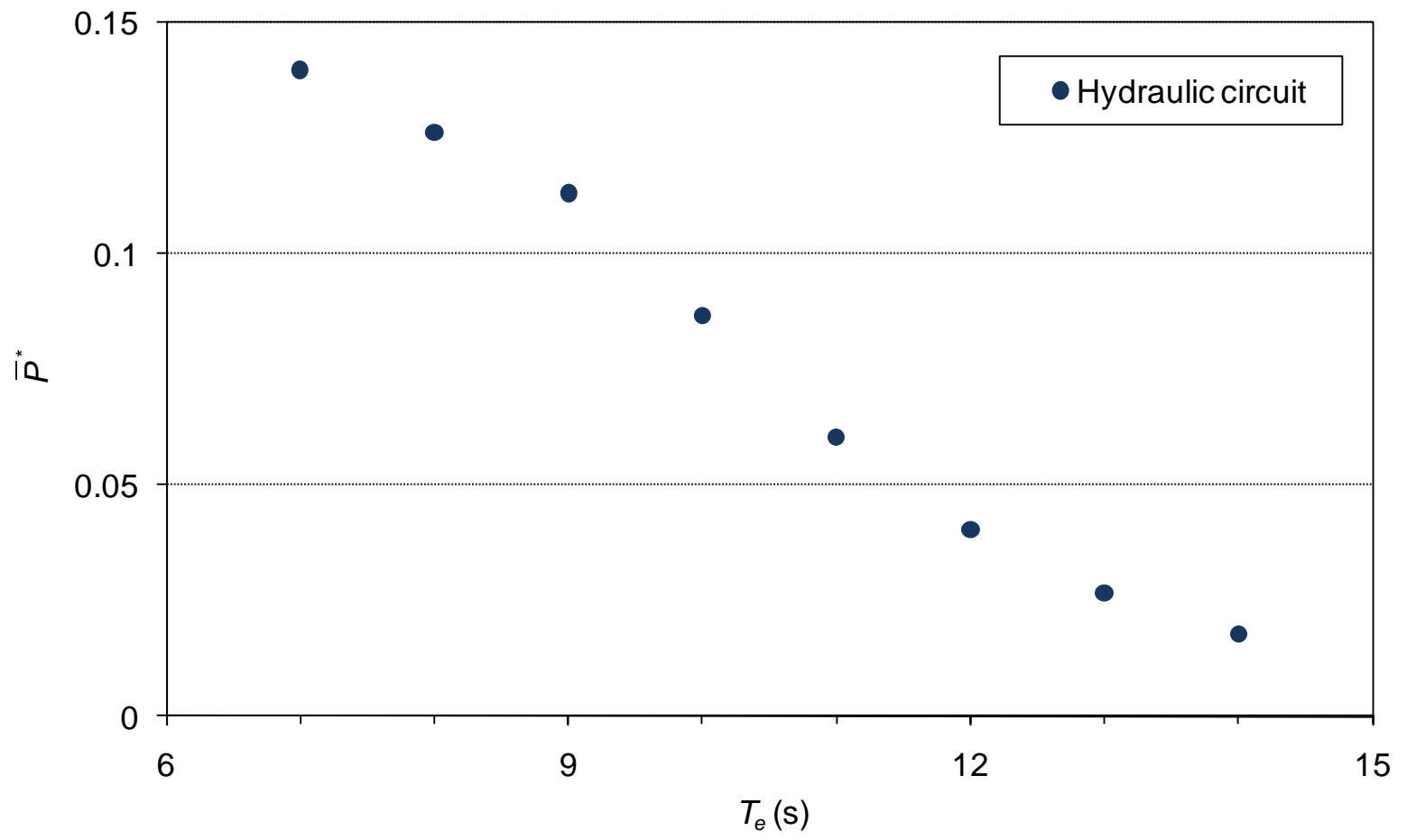




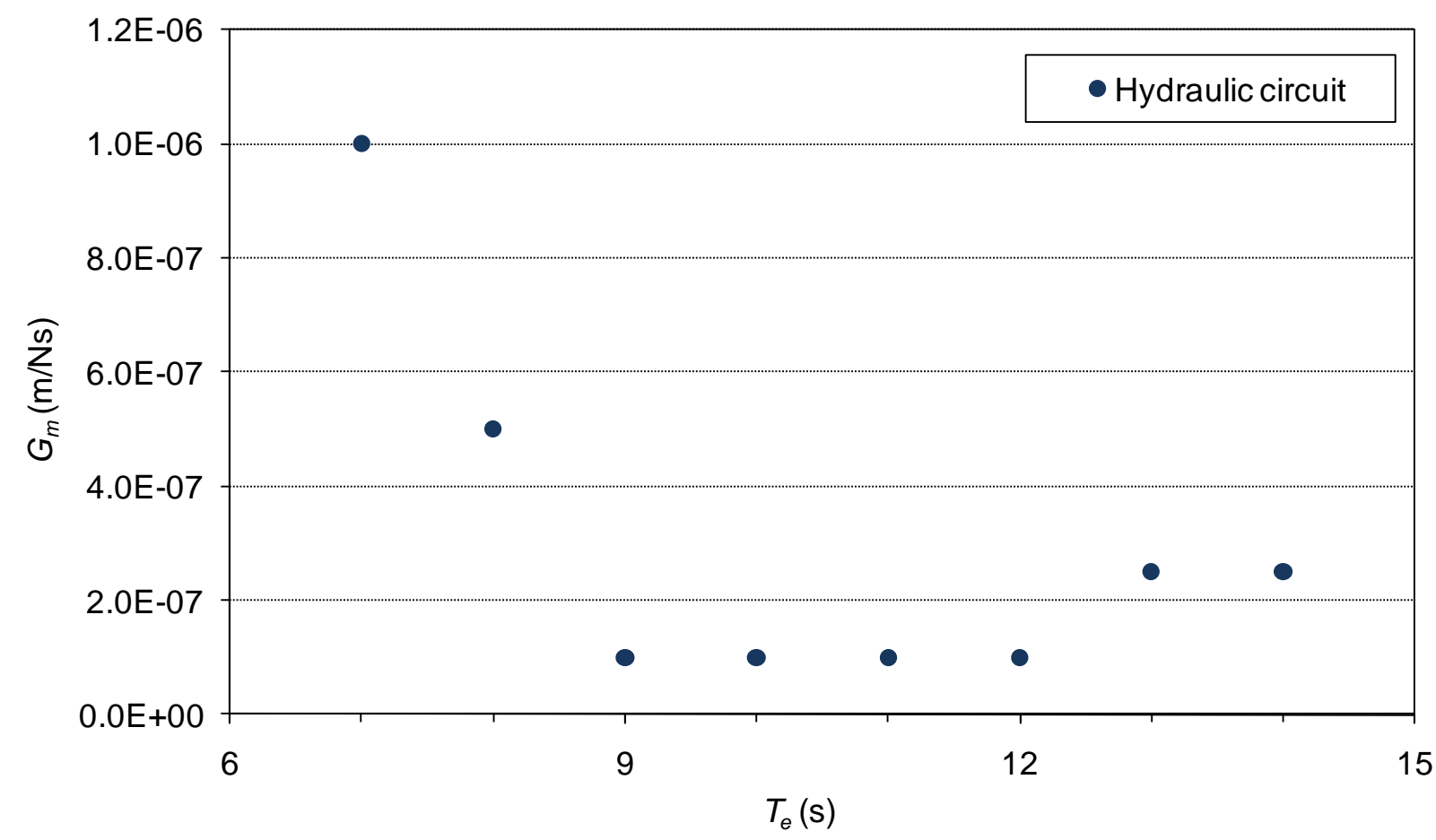


a

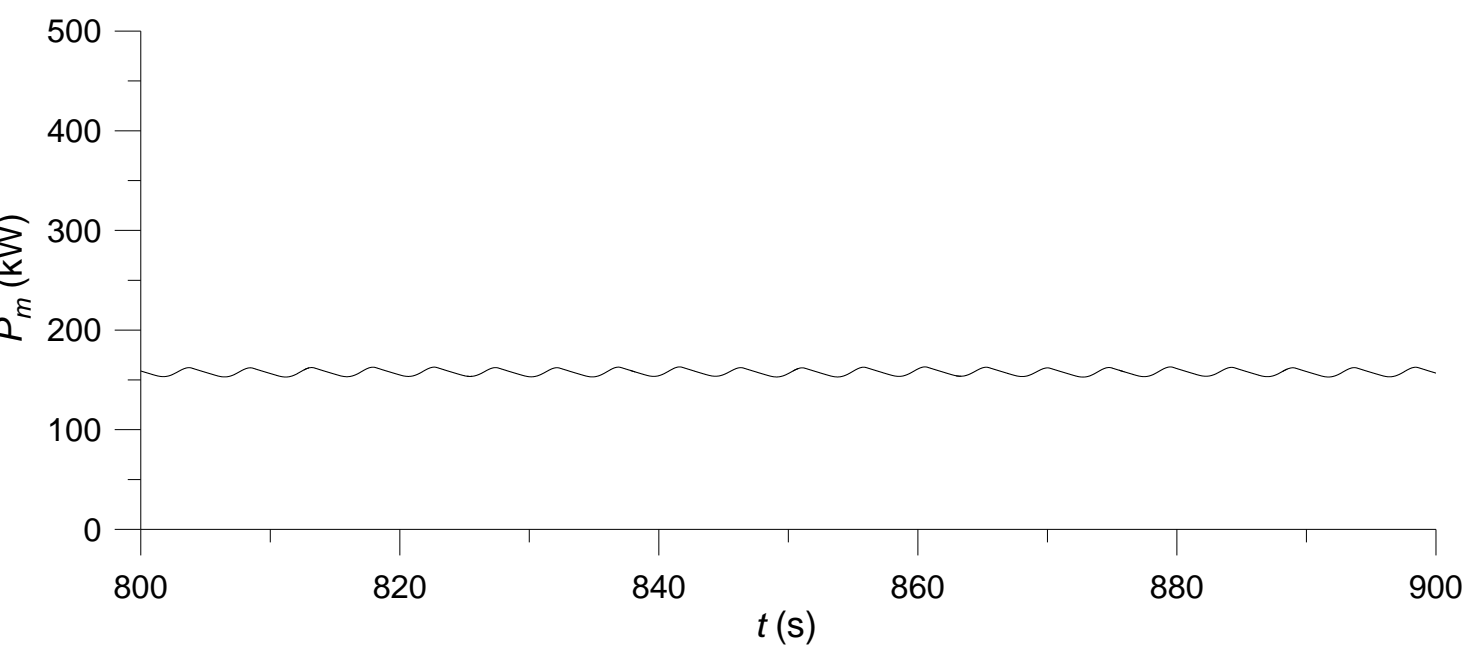

b

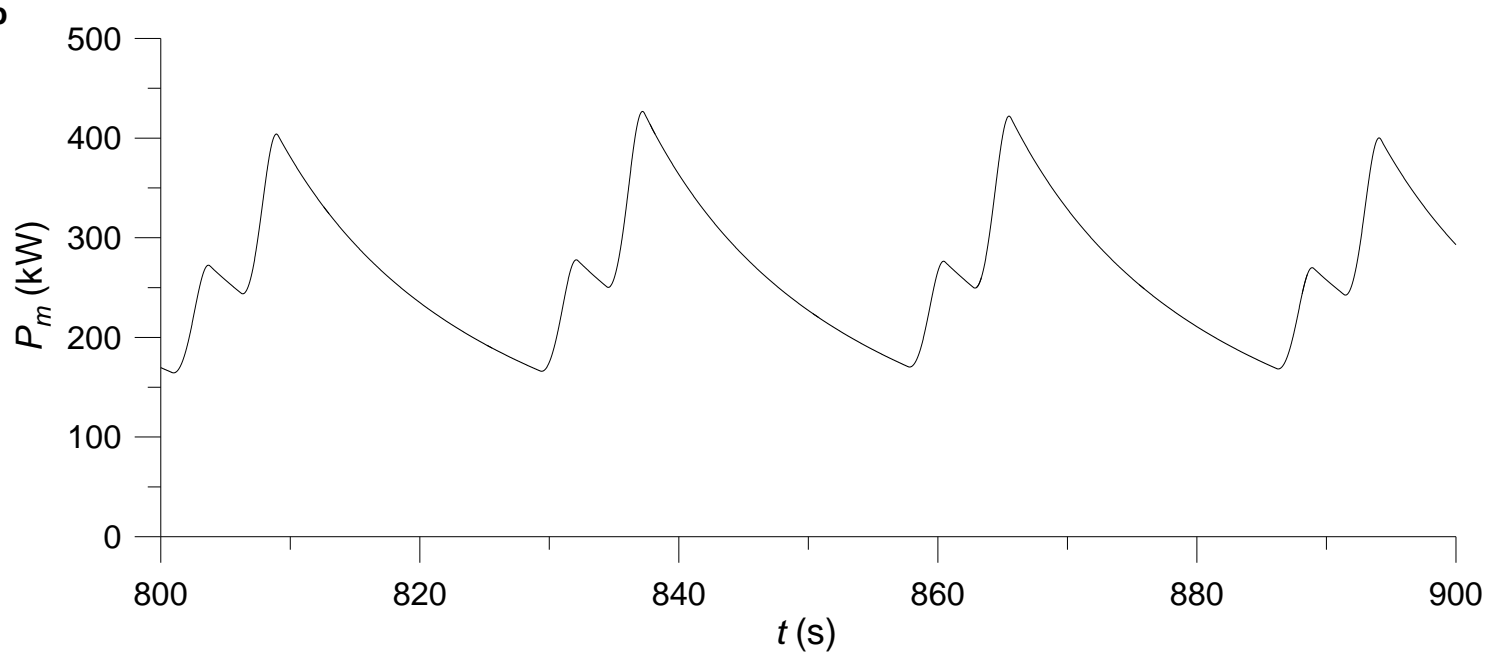


Figure13

a

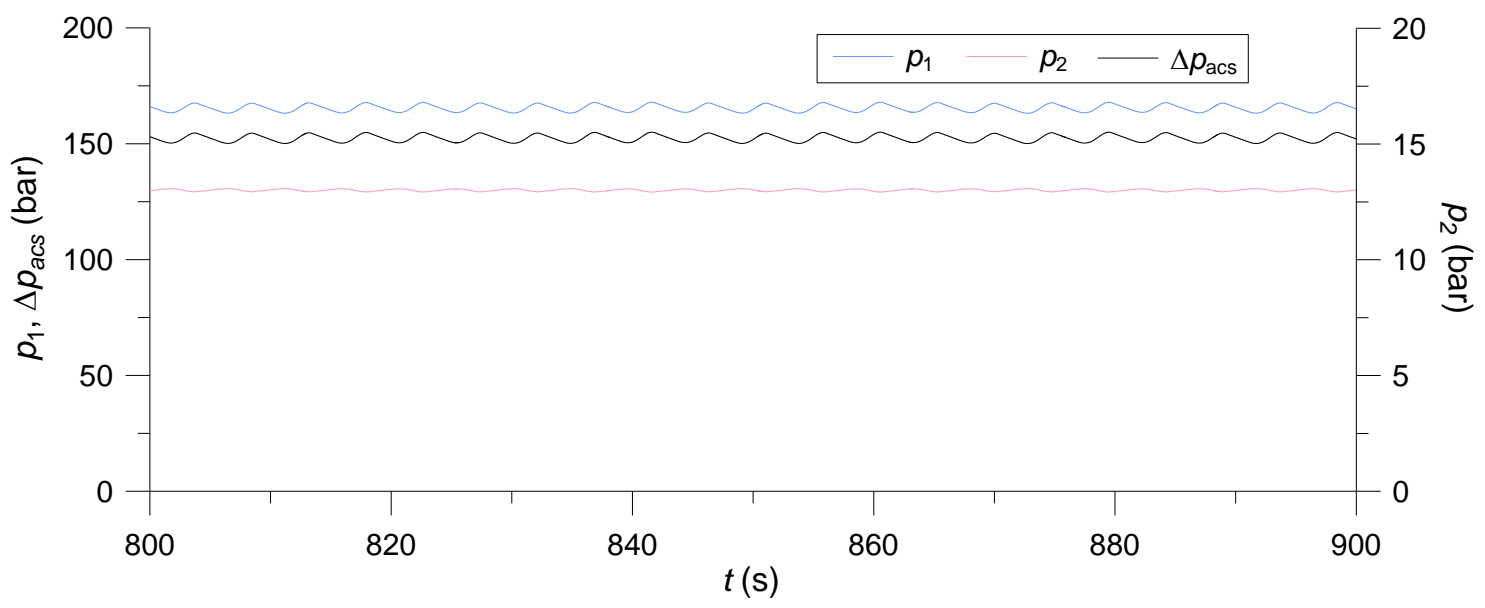

b

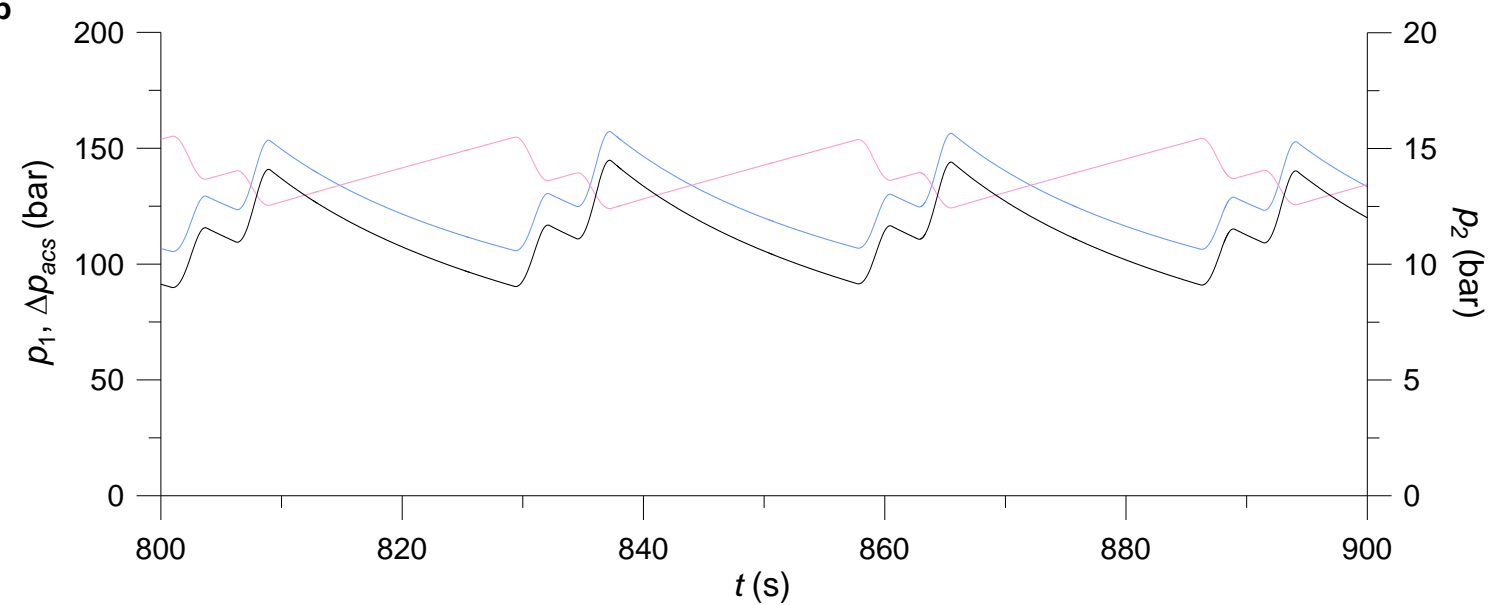




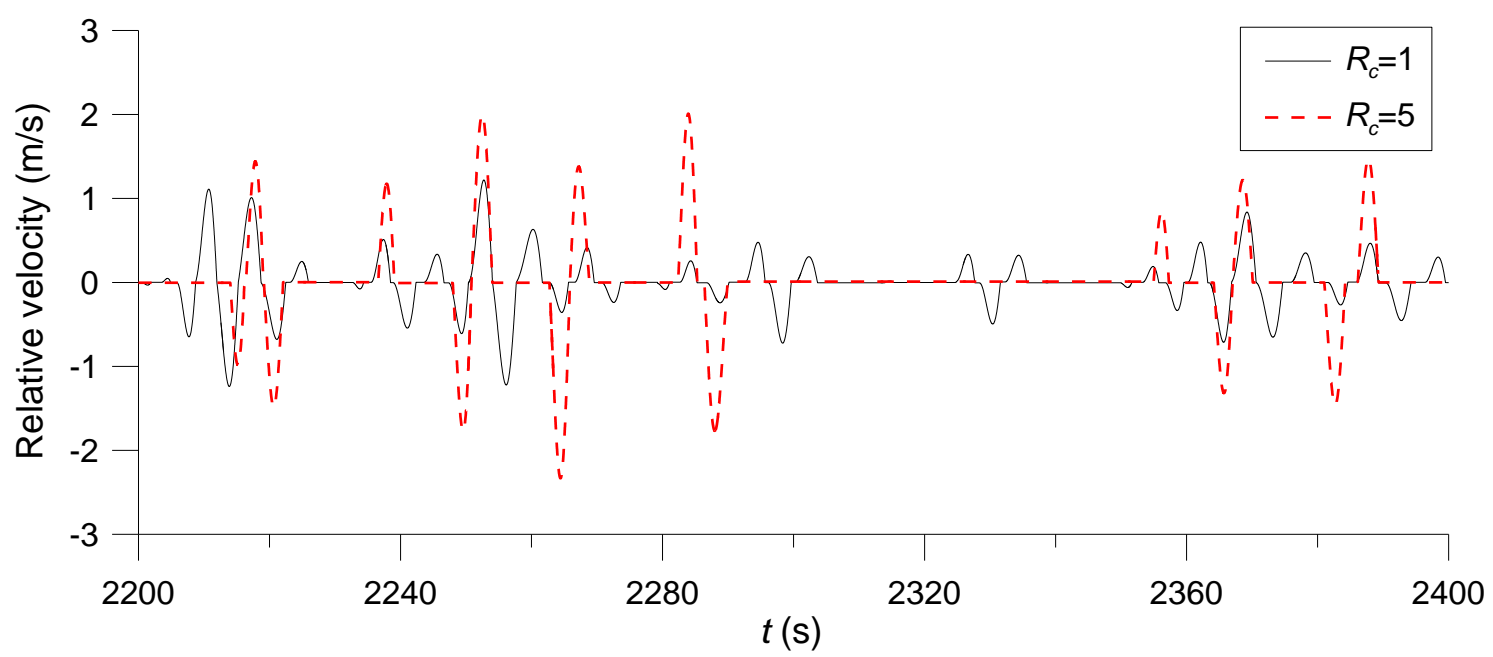


Figure15

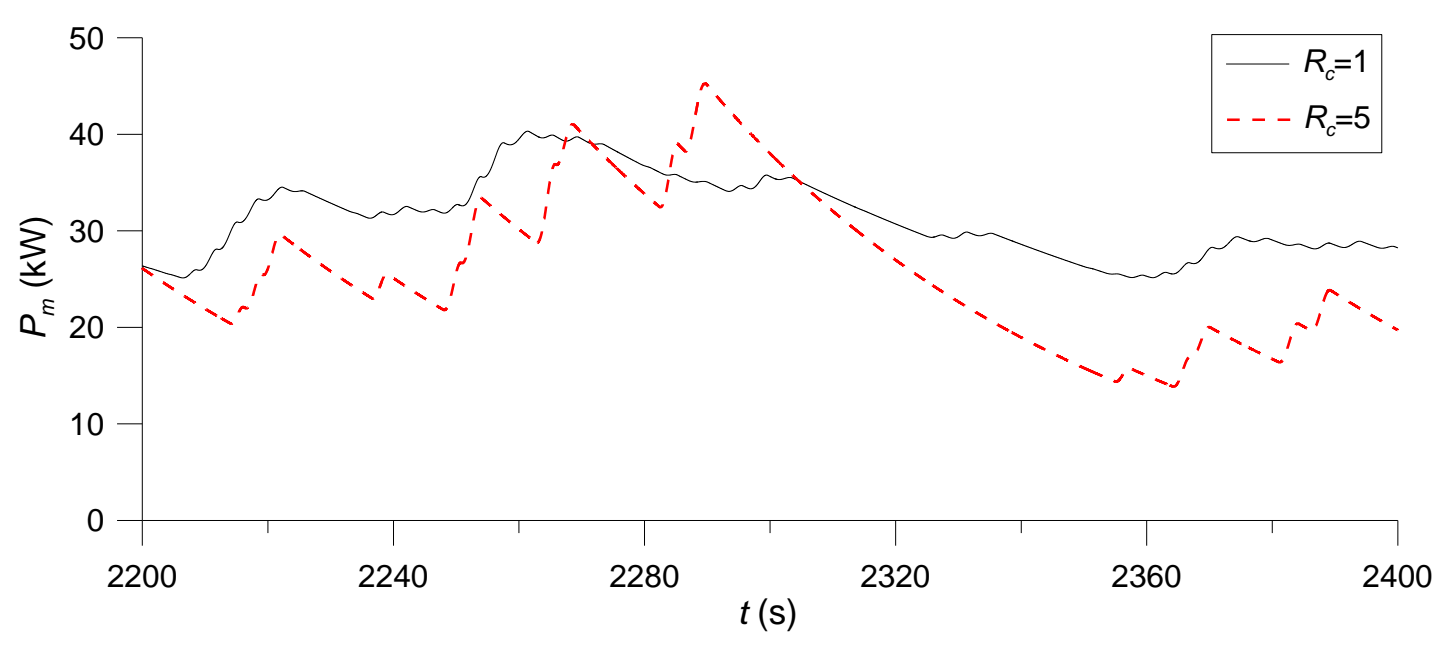




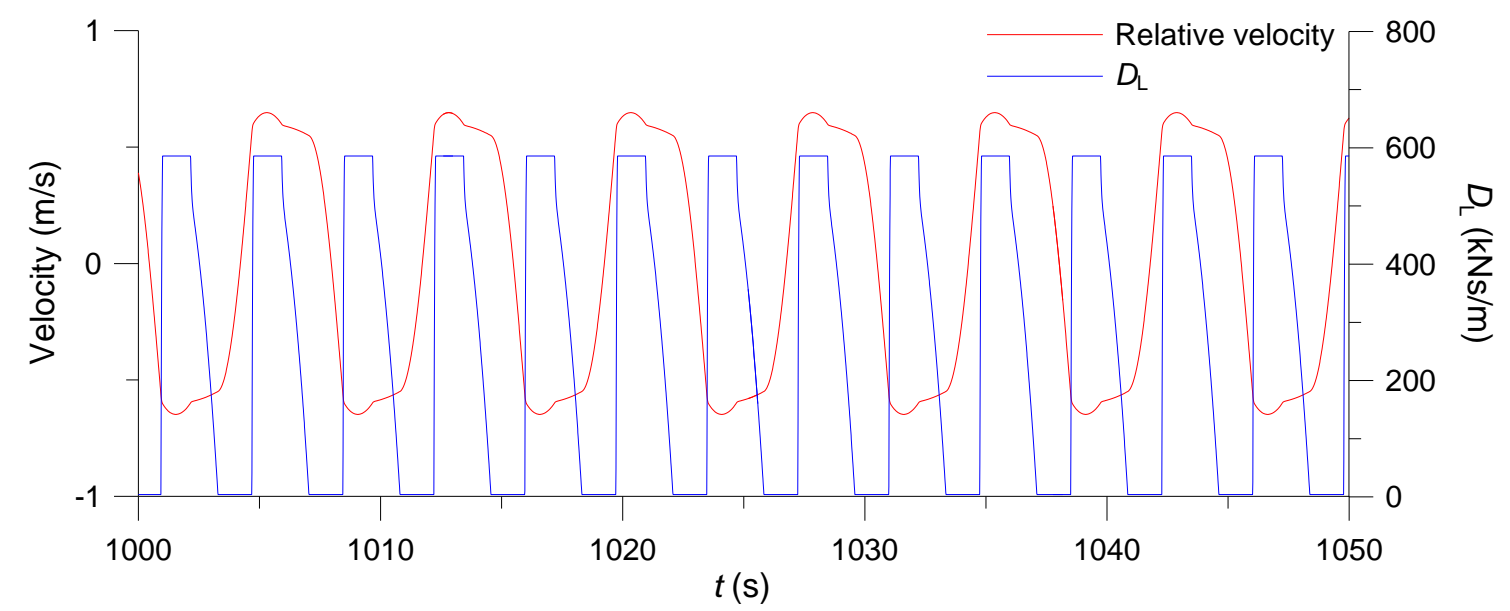


Figure17

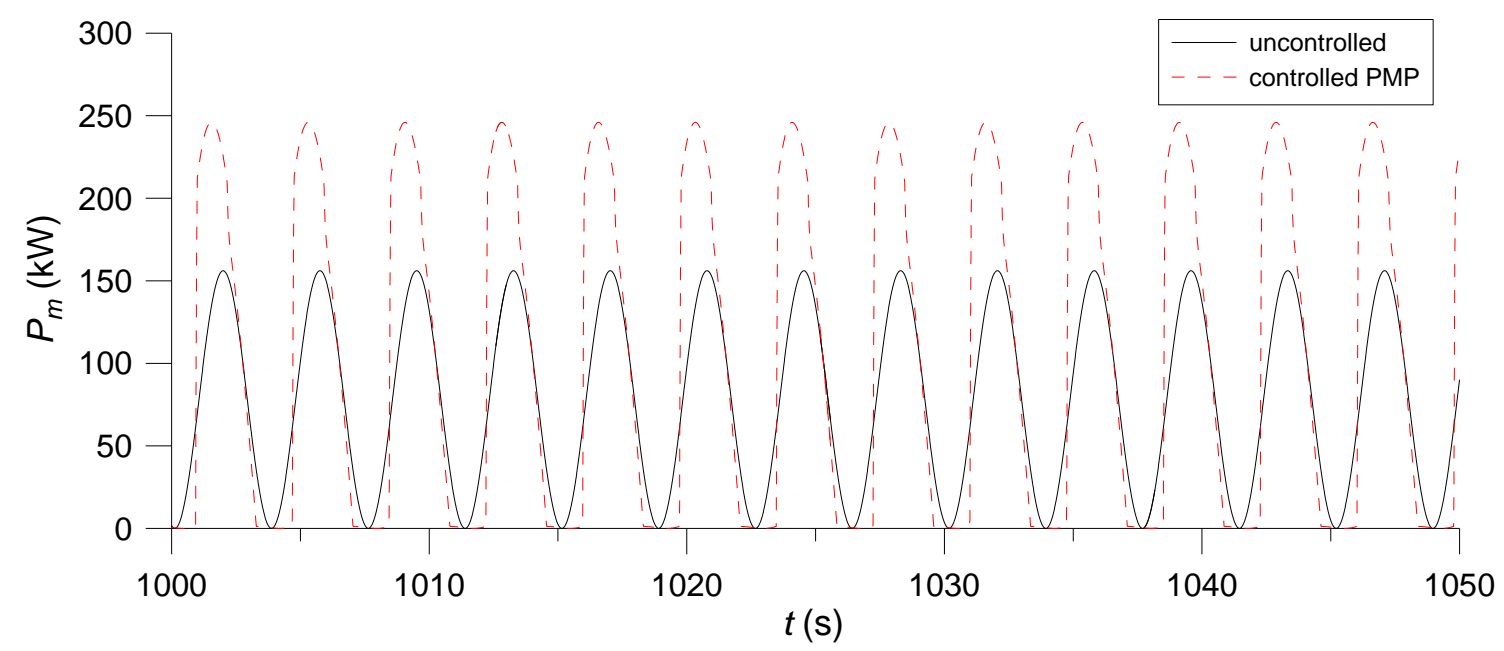




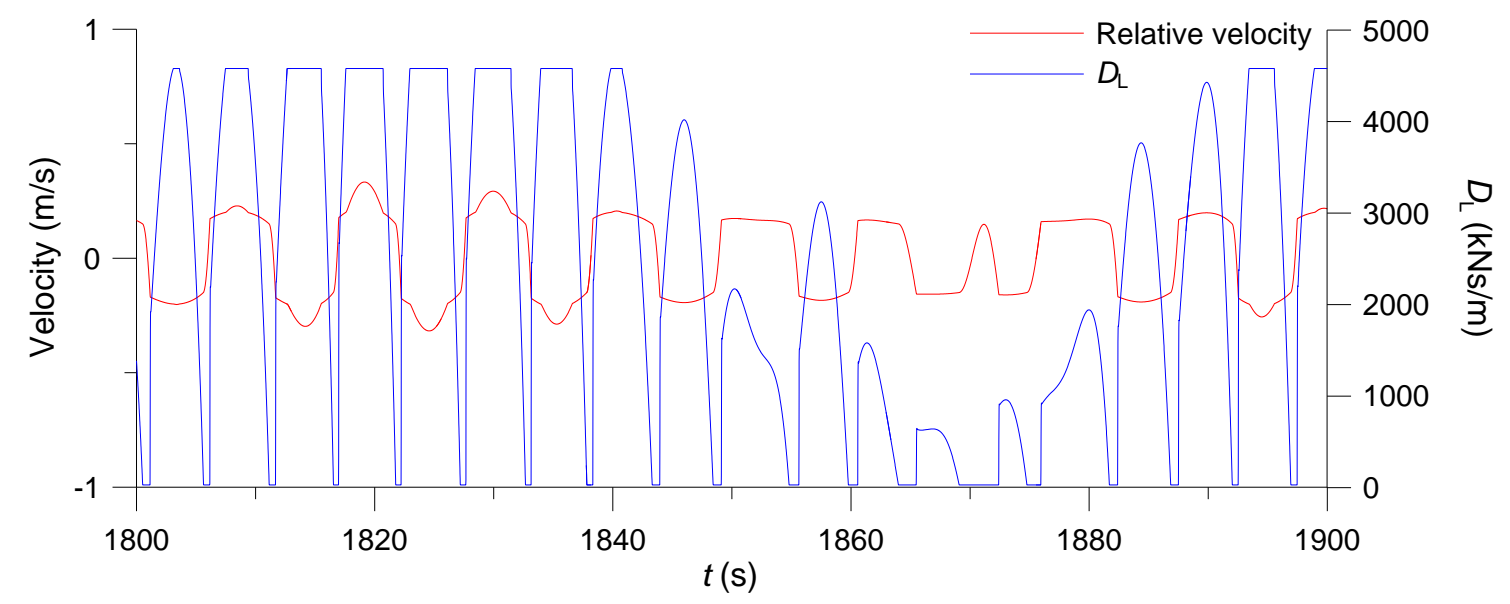


Figure19

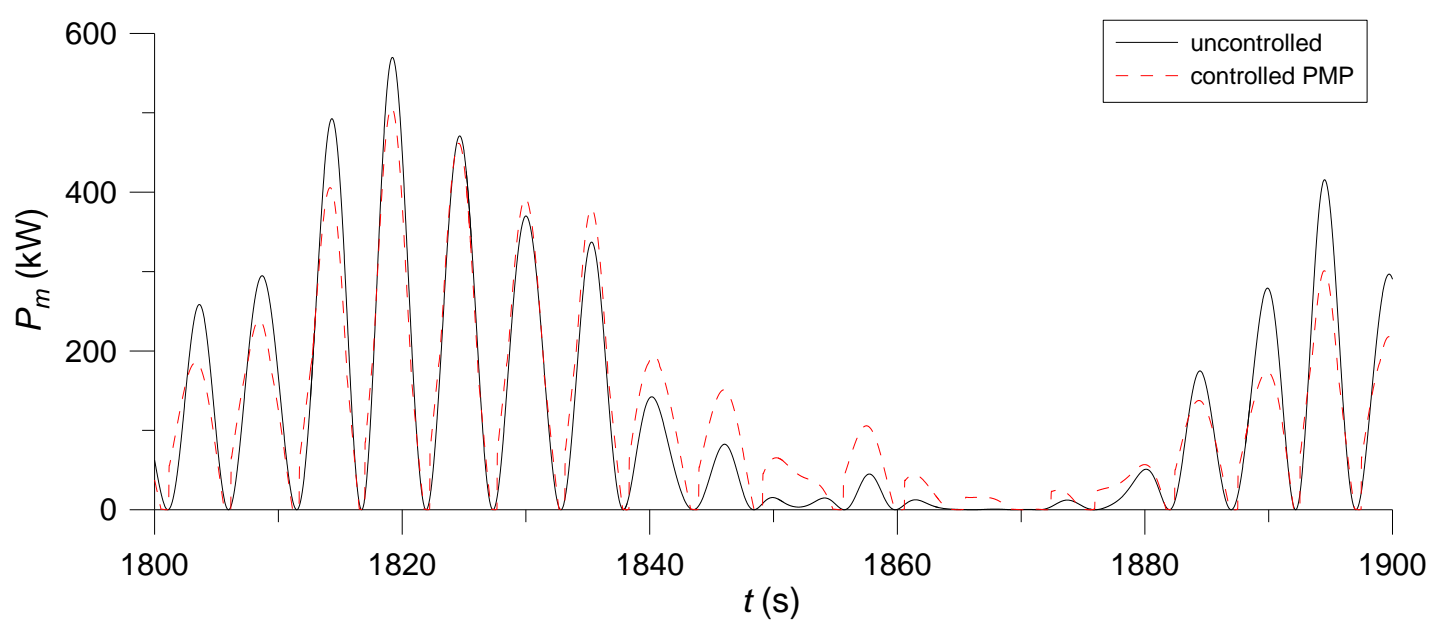

$6007 \quad\left[\begin{array}{l}\text { uncontrolled } \\ -\ldots \text { controlled PMP }\end{array}\right.$ 


\begin{tabular}{|c|c|c|c|c|}
\hline $\mathbf{L}_{\mathrm{L}}(\mathbf{k N s} / \mathbf{m})$ & $\begin{array}{c}\left|\dot{\xi}_{2}(t)-\dot{\xi}_{1}(t)\right|_{\text {inf }} \\
(\mathbf{m} / \mathbf{s})\end{array}$ & $\begin{array}{c}\left|\dot{\xi}_{2}(t)-\dot{\xi}_{1}(t)\right|_{\text {sup }} \\
(\mathbf{m} / \mathbf{s})\end{array}$ & $\boldsymbol{T}(\mathbf{s})$ & $\bar{P}(\mathbf{k W})$ \\
\hline $\begin{array}{c}390.7 \\
(\text { optimum) }\end{array}$ & - & - & 7.5 & 77.9 \\
\hline $\begin{array}{c}\text { Min=3.9 } \\
\text { Max=586.0 }\end{array}$ & 0.55 & 0.60 & 7.5 & 105.3 \\
\hline $\begin{array}{c}632.1 \\
\text { (optimum) }\end{array}$ & - & - & 8.5 & 88.0 \\
\hline $\begin{array}{c}\text { Min=6.3 } \\
\text { Max=948.2 }\end{array}$ & 0.35 & 0.4 & 8.5 & 106.5 \\
\hline
\end{tabular}




\begin{tabular}{|c|c|c|c|c|c|}
\hline $\boldsymbol{D}_{\mathrm{L}}(\mathbf{k N s} / \mathbf{m})$ & $\begin{array}{c}\left|\dot{\xi}_{2}(t)-\dot{\xi}_{1}(t)\right|_{\text {inf }} \\
(\mathbf{m} / \mathbf{s})\end{array}$ & $\begin{array}{c}\left|\dot{\xi}_{2}(t)-\dot{\xi}_{1}(t)\right|_{\text {sup }} \\
(\mathbf{m} / \mathbf{s})\end{array}$ & $H_{s}(\mathbf{m})$ & $T_{e}(\mathbf{s})$ & $\bar{P}(\mathbf{k W})$ \\
\hline $\begin{array}{c}2604.6 \\
(\text { optimum) }\end{array}$ & - & - & 2.0 & 9.0 & 52.8 \\
\hline $\begin{array}{c}\text { Min=260.5 } \\
\text { Max=3906.9 }\end{array}$ & 0.15 & 0.20 & 2.0 & 9.0 & 54.8 \\
\hline $\begin{array}{c}3053.5 \\
\text { (optimum) }\end{array}$ & - & - & 2.0 & 10.0 & 57.7 \\
\hline $\begin{array}{c}\text { Min=30.5 } \\
\text { Max=4580.3 }\end{array}$ & 0.15 & 0.20 & 2.0 & 10.0 & 59.9 \\
\hline
\end{tabular}

